US Army Corps

of Engineers ${ }_{\circledast}$

Engineer Research and

Development Center

Autonomous Navigation and Mapping in a Simulated Environment

Benjamin Christie, Osama Ennasr, and Garry Glaspell

September 2021 
The U.S. Army Engineer Research and Development Center (ERDC) solves the nation's toughest engineering and environmental challenges. ERDC develops innovative solutions in civil and military engineering, geospatial sciences, water resources, and environmental sciences for the Army, the Department of Defense, civilian agencies, and our nation's public good. Find out more at www.erdc.usace.army.mil.

To search for other technical reports published by ERDC, visit the ERDC online library at http://acwc.sdp.sirsi.net/client/default. 


\section{Autonomous Navigation and Mapping in a Simulated Environment}

Benjamin Christie, Osama Ennasr, and Garry Glaspell

Geospatial Research Laboratory

U.S. Army Engineer Research and Development Center

7701 Telegraph Road

Alexandria, VA 22315

Final report

Approved for public release; distribution is unlimited.

Prepared for U.S. Army Corps of Engineers

Washington, DC 20314-1000

Under Tactical Geospatial Information Capabilities (TGIC)

Program Element Number 622146

Project Number AT9

Task Number 01 


\section{Abstract}

Unknown Environment Exploration (UEE) with an Unmanned Ground Vehicle (UGV) is extremely challenging. This report investigates a frontier exploration approach, in simulation, that leverages Simultaneous Localization And Mapping (SLAM) to efficiently explore unknown areas by finding navigable routes. The solution utilizes a diverse sensor payload that includes wheel encoders, three-dimensional (3-D) LIDAR, and Red, Green, Blue and Depth (RGBD) cameras. The main goal of this effort is to leverage frontier-based exploration with a UGV to produce a $3-\mathrm{D}$ map (up to $10 \mathrm{~cm}$ resolution). The solution provided leverages the Robot Operating System (ROS).

DISCLAIMER: The contents of this report are not to be used for advertising, publication, or promotional purposes. Citation of trade names does not constitute an official endorsement or approval of the use of such commercial products. All product names and trademarks cited are the property of their respective owners. The findings of this report are not to be construed as an official Department of the Army position unless so designated by other authorized documents. 


\section{Contents}

Abstract........................................................................................................................................... if

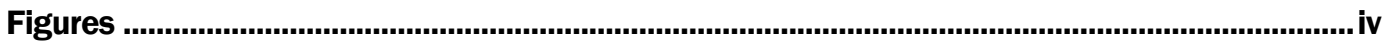

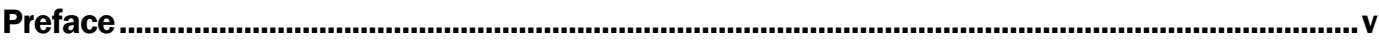

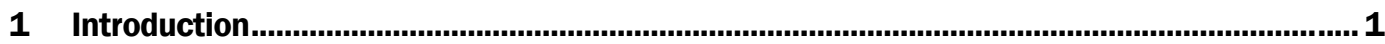

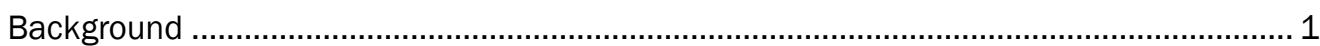

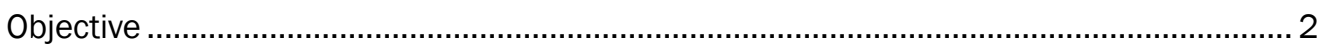

Overview of approach .............................................................................................. 3

2 Bringup and Robot Odometry ............................................................................................ 5

3 Accurate Mapping using SLAM Toolbox......................................................................... 7

Laser_assembler .................................................................................................... 9

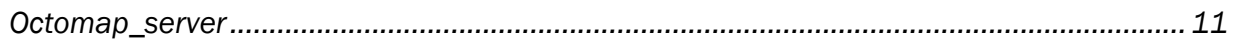

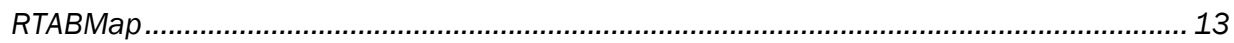

4 Path planning using Move_base and STVL ................................................................19

5 Autonomous Frontier-based Exploration using Explore Lite ............................................21

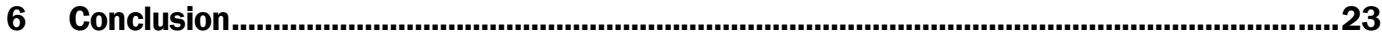

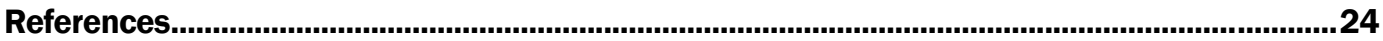

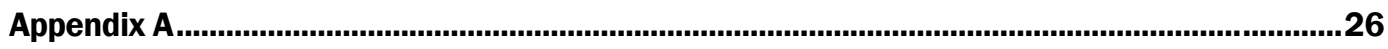

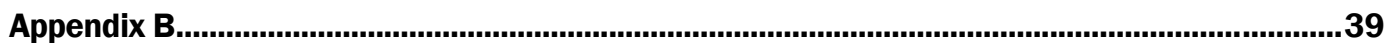

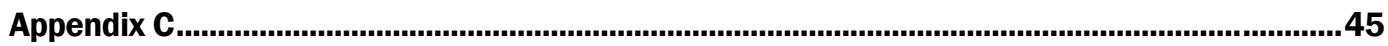

Appendix D............................................................................................................................50

Report Documentation Page 


\section{Figures}

Figure 1. Modified turtlebot3 with 3-D LIDAR and front and rear RGBD cameras loaded in the willow garage world (left). Odometry generated from the turtlebot3 compared to ground truth (right)

Figure 2. 2D occupancy grid of willow garage generated by SLAM toobox.

Figure 3. 3-D point cloud generated using the SLAM Toolbox in localization mode leveraging periodic_snapshotter.

Figure 4. 3-D point cloud generated using ocotomap_server...

Figure 5. 2-D occupancy grid generated from the SLAM Toolbox when fused with RTABMap.

Figure 6. 3-D point cloud of willow garage world generated by the SLAM Toolbox / RTABMap fusion.

Figure 7. 3-D point cloud of play pen world generated by the SLAM Toolbox / RTABMap fusion.

Figure 8. Costmap, with inflation layer, generated using the spatio_temporal_voxel_layer.

Figure 9. Frontier-based exploration using explore_lite over time. The frontiers are blue, and their associated costs are depicted by the green circles. The red dots depict the path of the robot and obstacles are marked with an inflation layer. 


\section{Preface}

This study was conducted for the U.S. Army Engineer Research and Development Center, Geospatial Research Laboratory (ERDC-GRL) under Program Element 622146/AT9/o1, "Tactical Geospatial Information Capabilities (TGIC)," Task "Tactical Data Generation and Processing." The technical monitor was Dr. Jean Nelson.

The work was performed by the Data Representation Branch of the Topography Imagery and Geospatial Research Division, ERDC-GRL. At the time of publication, Mr. Vineet Gupta was Branch Chief; Mr. Jeffrey Murphy was Division Chief; and Mr. Ritchie Rodebaugh was the Technical Director for Geospatial Research and Engineering (GRE) business area. The Deputy Director of ERDC-GRL was Ms. Valerie Carney and the Director was Mr. Gary Blohm.

The authors would like to acknowledge the following individuals for their contributions to this project: Dr. Jean Nelson, Mr. Vineet Gupta, Mr. Nathan Dodson, Mr. Mike Paquette, Mr. Ryan Kreiter and Mr. Tyler Howell.

The Commander of ERDC was COL Teresa A. Schlosser and the Director was Dr. David W. Pittman. 


\section{Introduction}

\section{Background}

Unknown Environment Exploration (UEE) (Wang et al. 2016; Roman et al. 2019) in the Robot Operating System (ROS) typically works in tandem with a map generated by Simultaneous Localization And Mapping (SLAM) (Castellanos et al. 2001; Jacobson et al. 2018). While there are multiple ways to accomplish this, the most common approach is frontier-based exploration (Keidar and Kaminka 2014; Wan et al. 2011). In frontier-based exploration, the boundary between "known" and "unknown" points (a frontier) becomes a navigation goal. Also produced is the associated cost to clear the frontier. The path-planning node subscribes to the navigation goals and attempts to clear all goals while keeping the cost at a minimum. This process repeats for all existing frontiers and with the addition of new frontiers. The most common type of map used for analysis is a twodimensional occupancy grid. Consequently, successful autonomous frontier-based exploration in ROS is grounded in the following:

1. Accurate odometry from the robot

2. Accurate $2 \mathrm{D}$ occupancy grid from SLAM

3. An efficient path-planner (avoids obstacles and detects navigable terrain)

4. A ROS node that efficiently outputs navigation goals to the path-planner

In this report, focus is placed on the problem of the frontier-based exploration. Specifically, the team identified and fused ROS packages that address each requirement listed above (1-4) to ensure successful frontierbased exploration. The combination of these packages results in a novel solution that allows autonomous exploration of $2 \mathrm{D}$ environments in simulation. To accomplish this, ROS leverages a layered approach. At the bottom of the stack is static layer (map_layer), which is the 2D map produced by the robot. The second layer (obstacle_layer) is comprised of obstacles detected by the sensors and is overlaid onto the static layer. The third layer (inflation_layer) generates a buffer around the obstacles as a safety measure to keep the robot from running into them. Finally, the combination of all layers refers to a costmap. 
It is worth noting that the default obstacle_layer generated by costmap2d (a ROS package that generates occupancy grids) ${ }^{1} 9$ creates persistent occupancies that function well in static environments. However, in a dynamic environment, the default obstacle_layer requires the sensors observation path to pass through the occupied space, which can fail to fully clear the moving object from the costmap resulting in phantom obstacles. This issue is especially noticeable when a person walks by the robot or when a door, that was previously closed, is opened. These, and other, issues make navigation in dynamic environments challenging. Therefore, also investigated was a spatio-temporal plugin to costmap2d designed specifically for dynamic environments by removing occupied spaces from the costmap if they are not re-observed after a set time.

\section{Objective}

This report addresses the Army Warfighting Challenges 2015 and the Army Robotic and Autonomous System (RAS) near-term objective of "Enhancing stand-off from threats and improve situation awareness" and their mid-term objective to "Enhance Platoon, Squad, and Soldier situation awareness." At a theatre level (CNA 17-21), the Army Critical Capability Gap(s) specifically list the following items ${ }^{23}$ :

1. Gap \#500459: "The Army units at Theater level lacks the capacity to integrate host-nation or third nation security forces into friendly sustainment area and base security operations during decisive action $100 \%$ of the time"

2. Gap \#500915: "The Army at theater level lacks the capability under decisive operations to detect personnel, material and facilities that are subsurface down to 100 meters"

3. Gap \#500917: "The Army at theater level lacks the capability under decisive operations to maintain situational awareness of subsurface structure down to 100 meters"

4. Gap \#500918: "The Army at theater level lacks the capability under decisive operations to Map a subsurface structure as specified in the mission with Standard Shareable Geospatial.”

\footnotetext{
1 http://wiki.ros.org/costmap_2d

2 ArmyWarfightingChallenges.pdf

3 robototic_autonomous_systems_strategy.pdf (army.gov.au)
} 


\section{Overview of approach}

This report focuses on getting a robot, specifically a turtlebot $3,{ }^{1}$ to map autonomously in simulations leveraging both ROS Melodic ${ }^{2}$ and Gazebo 93. There are three models of the turtlebot 3 available, specifically the "burger," "waffle," and "waffle pi." Only the "waffle" is equipped with an Red, Green, Blue, and Depth (RGBD) camera, and as a result, used throughout the report. The original turtlebot 3 configuration was modified to include an additional rear RGBD camera and centrally mounted $3 \mathrm{D}$ LIDAR. The addition of a rear camera increases the frequency of loop closure and improves the accuracy of the SLAM-generated map.

The simulated turtlebot3 comes equipped with an odometry plugin. An additional plugin to track ground truth position of the robot was added to verify accuracy. The output of these plugins was compared with the odometry of the turtlebot3. Fortunately, the robot's odom matched that of the ground truth. This was not the case when other virtualized robots were tested. Ultimately, the turtlebot3's odom ensured the first requirement (R1) was satisfied and did not require modification.

For the second requirement (R2), the SLAM_toolbox ROS package was chosen4. SLAM_toolbox will be the defato standard in ROS 2.0. This package shows promise in generating accurate $2 \mathrm{D}$ occupancy grids and is discussed in length later in this report. As a 2D SLAM approach, it does not inherently produce 3-D point clouds of the mapped environment. Therefore, additional ROS packages were incorporated to produce 3-D point clouds to increase functionality.

To satisfy the third requirement (R3), the team down selected from a list of global and local path planners. For global planners, the three most common path planners are carrot planner 5 , nav2 $\mathrm{fn}^{6}{ }^{6}$, and global_planner7. For this study, the global_planner was chosen because it allowed for tuning of the variables "neutral cost," "lethal cost," and "cost factor."

\footnotetext{
1 https://emanual.robotis.com/docs/en/platform/turtlebot3/overview/

2 http://wiki.ros.org/melodic

3 http://gazebosim.org/

4 https://github.com/SteveMacenski/slam_toolbox

5 http://wiki.ros.org/carrot planner

6 http://wiki.ros.org/navfn

7 http://wiki.ros.org/global_planner
} 
Neutral cost is the value assigned to open space and lethal cost assigned to obstacles. The cost factor is used to spread cost values evenly over the output range so free space and lethal obstacles are well separated. The package global_planner also allows to choose between A/* or Dijkstra algorithms to produce paths. As for local planners, Dynamic Window Approach (DWA) ${ }^{1}$, Elastic Band (EB) ${ }^{2}$, and Timed Elastic Band (TEB) 3 are the most common implementations. EB was chosen since it is not as computationally as expensive as TEB and the team has observed the robot unable to move, on more than one occasion, when using DWA.

In ROS Kinetic, there are many options for frontier-based exploration, as noted in Glaspell et al. (2020). However, in a newer distribution such as ROS Melodic, there are significantly less options for frontier-based exploration. The aforementioned packages either do not compile on newer ROS distributions or do not function as intended. To satisfy the fourth requirement (R4), the ROS package explore_lite 4 was utilized for autonomous frontier-based exploration. In the remainder of this report, each step of this approach will be discussed in more detail and how it addresses the requirements for frontier-based exploration.

1 http://wiki.ros.org/dwa local_planner

2 http://wiki.ros.org/eband local planner

3 http://wiki.ros.org/teb_local_planner

4 http://wiki.ros.org/explore_lite 


\section{Bringup and Robot Odometry}

The turtlebot3 comes pre-equipped with a forward facing RGBD camera and a $2 \mathrm{D}$ laser. The original model was modified to incorporate the rear camera and the $2 \mathrm{D}$ laser was replaced with a 3 -D LIDAR. The xacro configuration files for the modified turtlebot 3 and the launch file for the willow garage world are provided in Appendix A. The willow garage world was specifically chosen since it is one of the larger simulated worlds. To launch the willow garage world with the modified turtlebot 3 run the following command from the directory containing the launch file below:

terminal 1, launch world and robot: roslaunch

turtlebot3_willow_3d_2.launch

terminal 2, launch SLAM module: roslaunch tb3_online_sync_rtab

terminal 3, launch movebase: roslaunch

tb3_move_base_slambox_basic_EBLP.launch

terminal 4, launch explore lite: roslaunch explore_lite

explore_lite_gridmap.launch

After running the command in terminal 1, gazebo launches the willow garage world and the modified turtlebot3 (Figure 1). 
Figure 1. Modified turtlebot3 with 3-D LIDAR and front and rear RGBD cameras loaded in the willow garage world (left). Odometry generated from the turtlebot3 compared to ground truth (right).
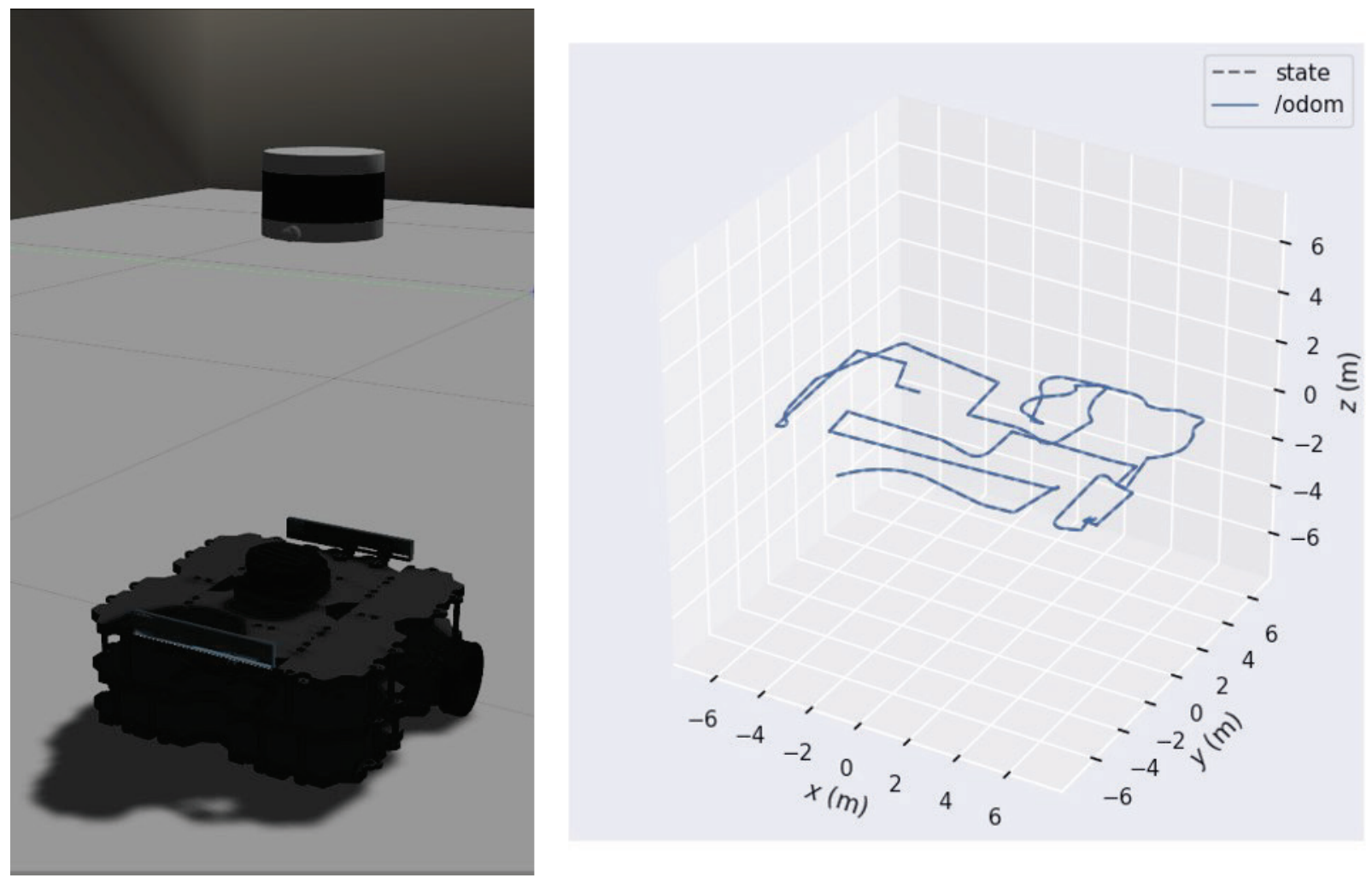

Figure 1 also shows the odometry from the turtlebot3 compared to the ground truth generated from the p3d_base_controller plugin. The odometry from the robot when compared to ground truth shows a near 1:1 correspondence, which is not always the case with simulated robots. Note that the accurate odometry satisfies the first requirement for successful frontier-based exploration. Provided below is the p3d_base_controller plugin code.

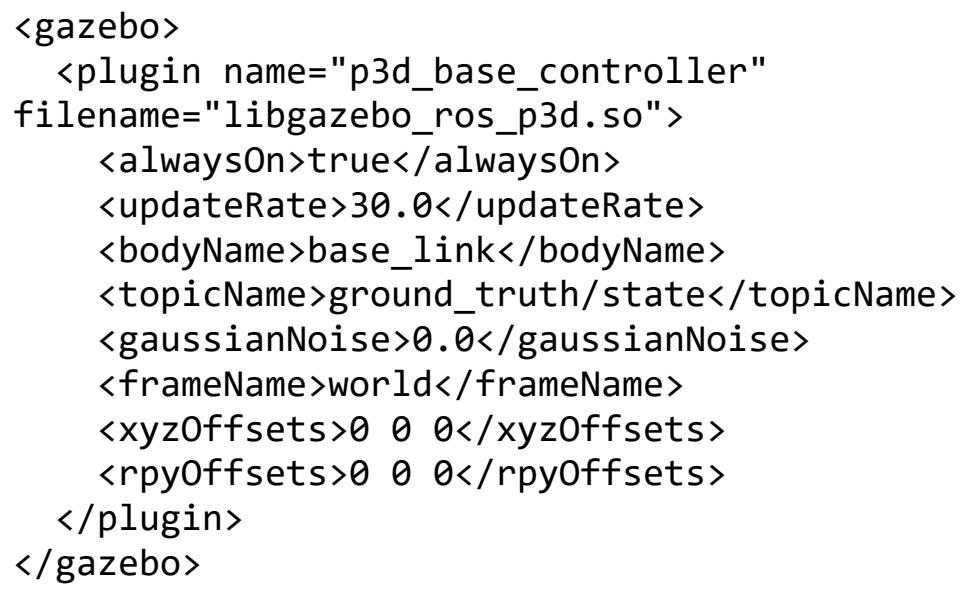




\section{Accurate Mapping using SLAM Toolbox}

At its core, successful frontier-based exploration relies heavily on SLAM for localization and navigation. If the SLAM-generated grid map is inaccurate, the robot will have difficulty avoiding obstacles and reaching its intended goal. In addition, phantom obstacles (obstacles that do not exist in reality but show in the cost map) can cause the path-planning software to fail. For this report, the team focused on the SLAM_toolbox built by Steve Macenski ${ }^{1}$. SLAM_toolbox is a 2D SLAM approach that has multiple modes of operation. In addition to localization and mapping modes, mapping can be broken down into synchronous (all scans are processed), asynchronous (not all scans are processed, but useful for large spaces $>200,000 \mathrm{ft}^{2}$ ), and lifetime mode. In this report, the team focused on synchronous mode, but lifetime mode is of particular interest since existing maps are updated with subsequent scans. Within the SLAM_toolbox configuration file, the ROS parameters are as follows:

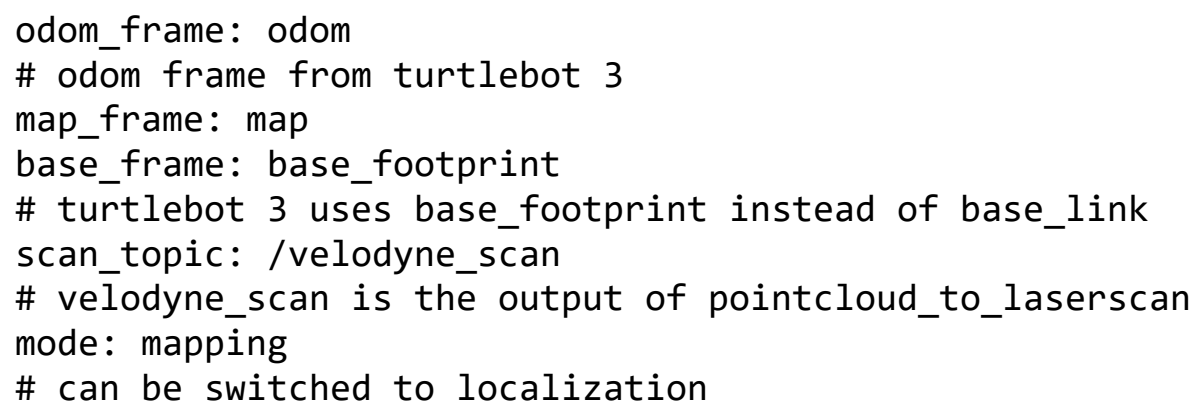

SLAM toolbox accepts a laser scan topic; however, in this report, the pointcloud_to_laserscan nodelet was leveraged, which converts a 3-D PointCloud2 message from the LiDAR to a 2D LaserScan message. In short, the pointcloud_to_laserscan node analyzes all points from the 3-D point cloud that overlap along the $\mathrm{z}$-axis and returns the point closet to the robot. SLAM toolbox uses this modified LaserScan message for grid_map construction and localization. Using pointcloud_to_laserscan to a compressed 3-D point cloud into 2D allows the robot to detect obstacles that a 2D laser scan device would typically miss while still mapping (and processing) in 2D. Shown below is an example of the pointcloud_to_laserscan nodelet launch.

\footnotetext{
1 https://github.com/SteveMacenski/slam_toolbox
} 


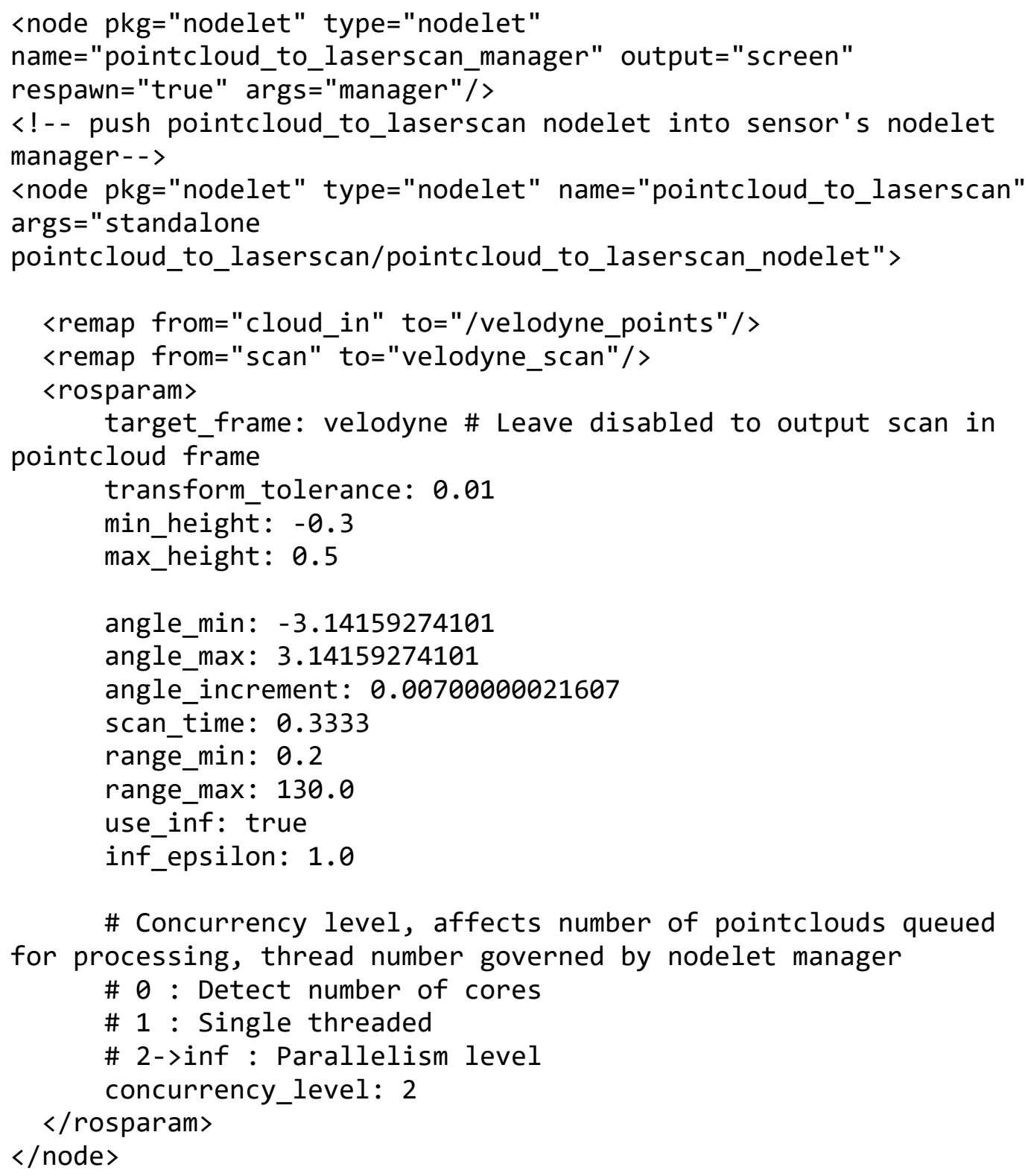

In this example, "cloud_in" is the output from the simulated velodyne LIDAR. The parameter "min_height" is with respect to the 3-D LIDAR frame and is set to $2 \mathrm{~cm}$ above ground level. This ensures the collapsed LaserScan topic does not clip the ground when moving. The "max_height" variable is set to the robot's height. The variables "angle_min" and "angle_max" are set for a 36o-degree LIDAR. The "angle_increment" parameter is $2^{*} \mathrm{pi} /($ number of horizontal laser points from LIDAR). In addition, "range_min" and "range_max" are set to match the velodyne's output. The nodelet outputs the topic velodyne_scan, which were passed to the SLAM toolbox. It is significant to note that SLAM toolbox does not produce $3-\mathrm{D}$ point clouds. Several attempts were made to integrate $3-\mathrm{D}$ 
pointclouds with varying degrees of success. Specifically, the attempts were made with laser_assembler ${ }^{1}$, ocotomap_server ${ }^{2}$, and RTABMap3.

\section{Laser_assembler}

The first attempt leveraged the nodes laser_assembler and periodic_snapshotter. Note that the periodic_snapshotter node requires compiling laser_assembler from source. The sample code to launch these is provided below.

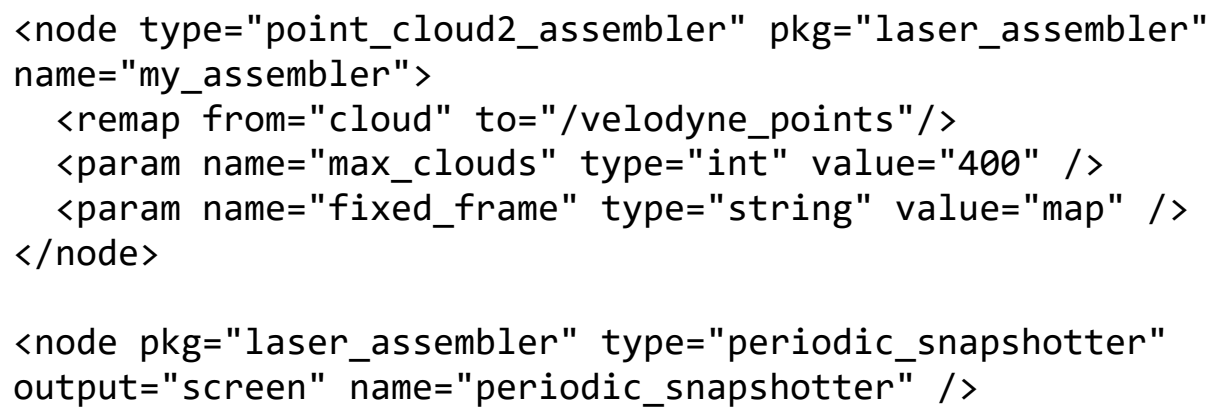

In this setup, the laser_assembler node builds a queue of collected PointCloud2 messages, $\sim 400$, and the periodic_snapshotter writes the queue to a file every 10 seconds. While this allowed the team to generate a 3 -D point cloud as it mapped, there was a significant drawback to this approach. Specifically, loop closure does not correct point clouds already written to the file. Thus, any drift that occurred before loop closure is apparent when viewing the files in CloudCompare. To work around this limitation with periodic_snapshotter, two runs were made in the willow garage. The first run used mapping mode to generate a serialized map, which can be seen in Figure 2. Due to successful loop closure, the resulting map does not possess any double walls, the wall thickness is uniform, and opposite walls are parallel. Any incurred drift over the $900 \mathrm{~m}$ mapped area was negligible. It is significant to note that the occupancy grid generated from the SLAM Toolbox outperforms other SLAM modules regarding accuracy and fully satisfies the second requirement for successful frontierbased exploration.

\footnotetext{
1 https://github.com/ros-perception/laser assembler

2 https://github.com/OctoMap/octomap_mapping

3 https://github.com/introlab/rtabmap_ros
} 
Figure 2. 2D occupancy grid of willow garage generated by SLAM toobox.

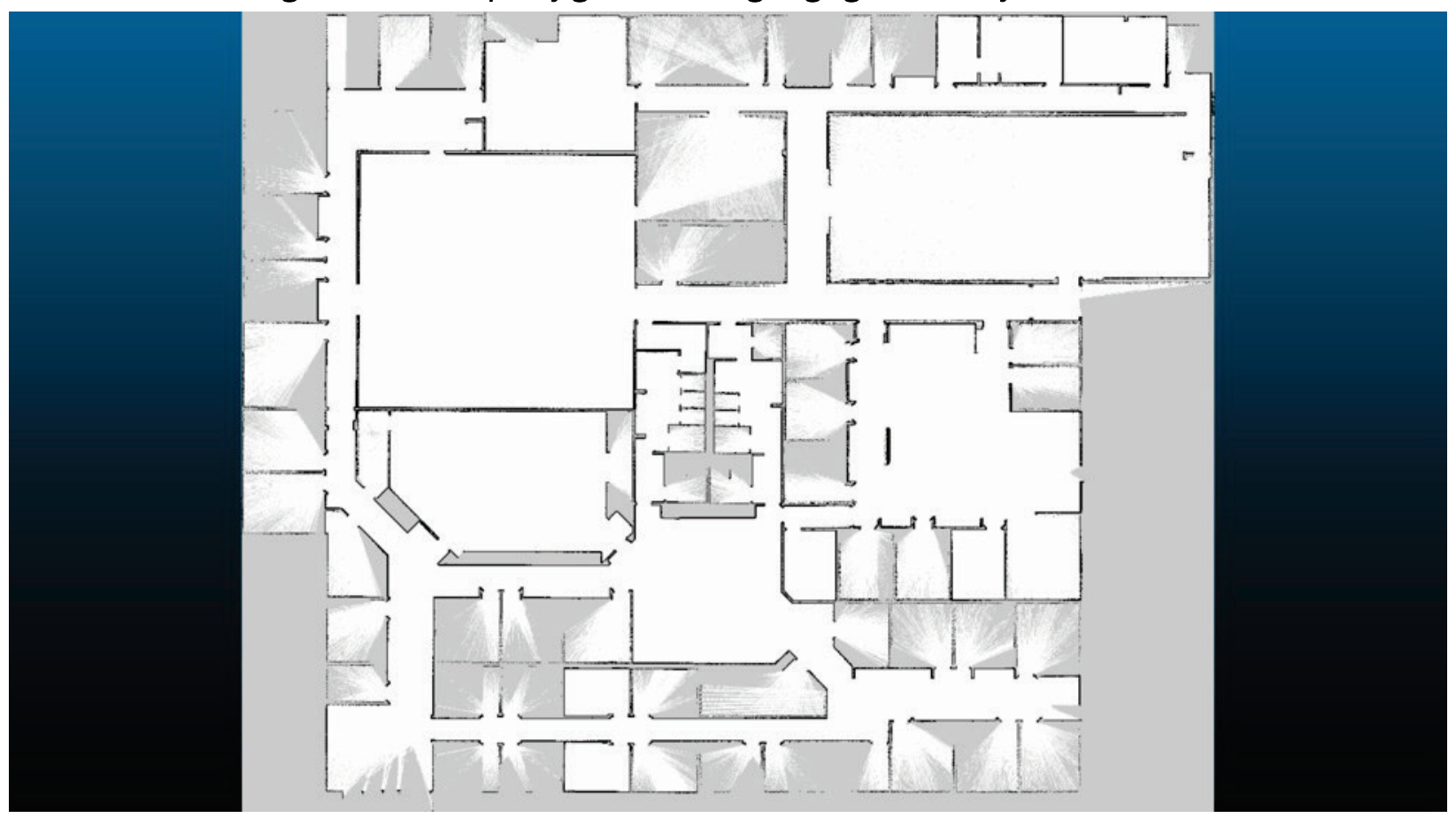

To generate the 3-D pointcloud with periodic_snapshotter, a second run was made with the SLAM Toolbox in localization mode. This approach was quite effective, as can be seen in Figure 3. However, in large areas, this approach may not be realistic due to battery limitations of the robot or the time required making two complete runs. Limitations aside, the resulting 3-D map also showed negligible drift like the 2-D map. 
Figure 3. 3-D point cloud generated using the SLAM Toolbox in localization mode leveraging periodic_snapshotter.

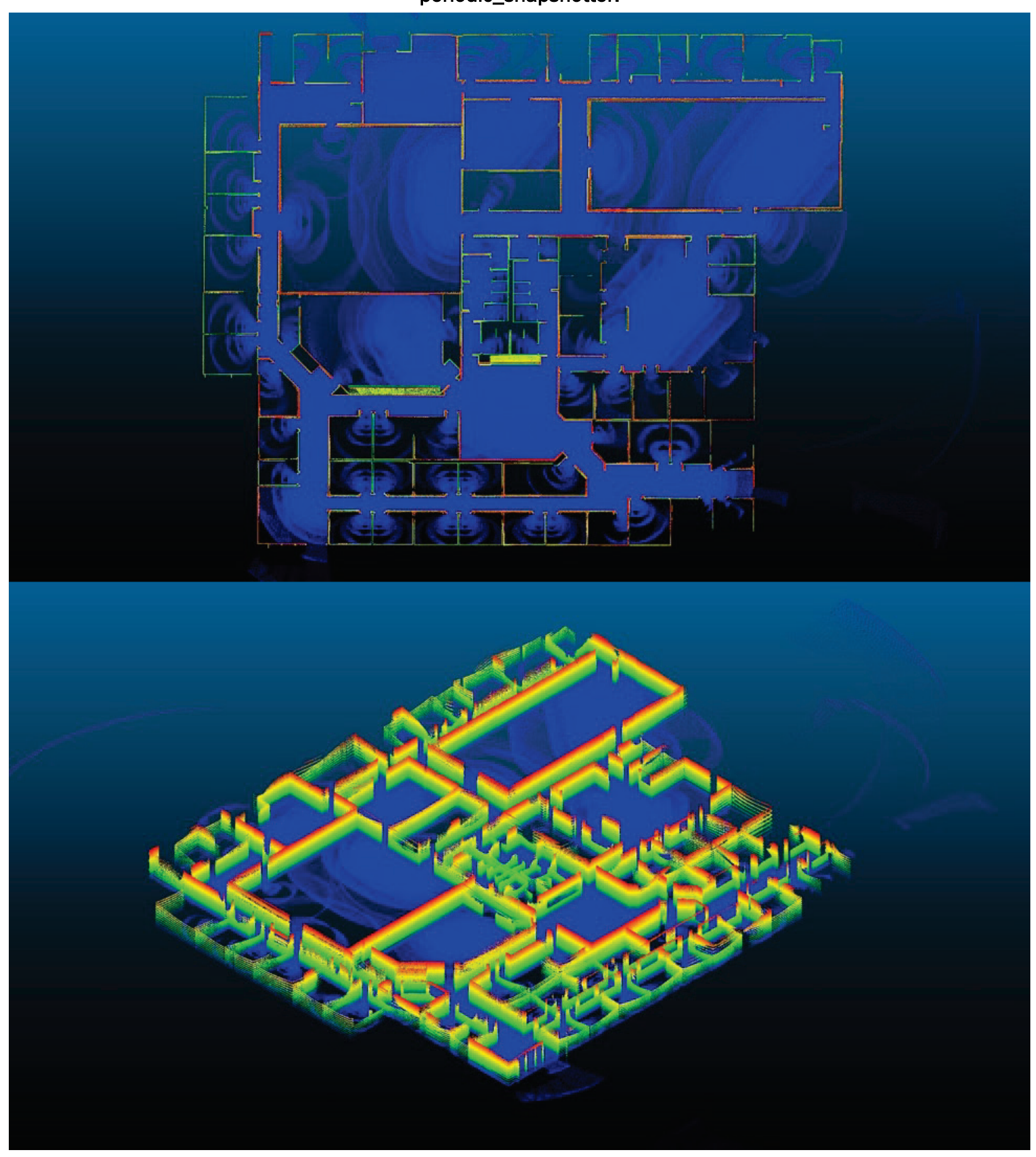

\section{Octomap_server}

The second attempt leveraged ocotomap_server to generate a $3-\mathrm{D}$ point cloud. The main advantage of octomap_server is that it only required a single mapping run, in contrast to the two runs required by the 
periodic_snapshotter approach. Since the point cloud was exported at end of the run, all loop closures were accounted for. One drawback was noticed while using ocotmap server; more RAM was consumed the longer octomap server ran. The base configuration, without octomap_server running, typically consumes $\sim 4.3 \mathrm{~GB}$ of RAM. After mapping half of willow garage, RAM usage was $>6 \mathrm{~GB}$ and when nearly done it was $>8 \mathrm{~GB}$. On our physical robot, the current onboard computer only has $8 \mathrm{~GB}$ of RAM so this could be a potential issue for long scans. It is possible to reduce RAM consumption by decreasing the resolution of the 3-D pointcloud in octomap_server. Shown below is the sample code for octomap_server node. Also shown below in Figure 4 is the 3 -D point cloud generated by octomap_server. Like the previous approach, the octomap_server produced a 3-D point cloud that did not have double walls, the wall thickness is uniform, and opposite walls are parallel.

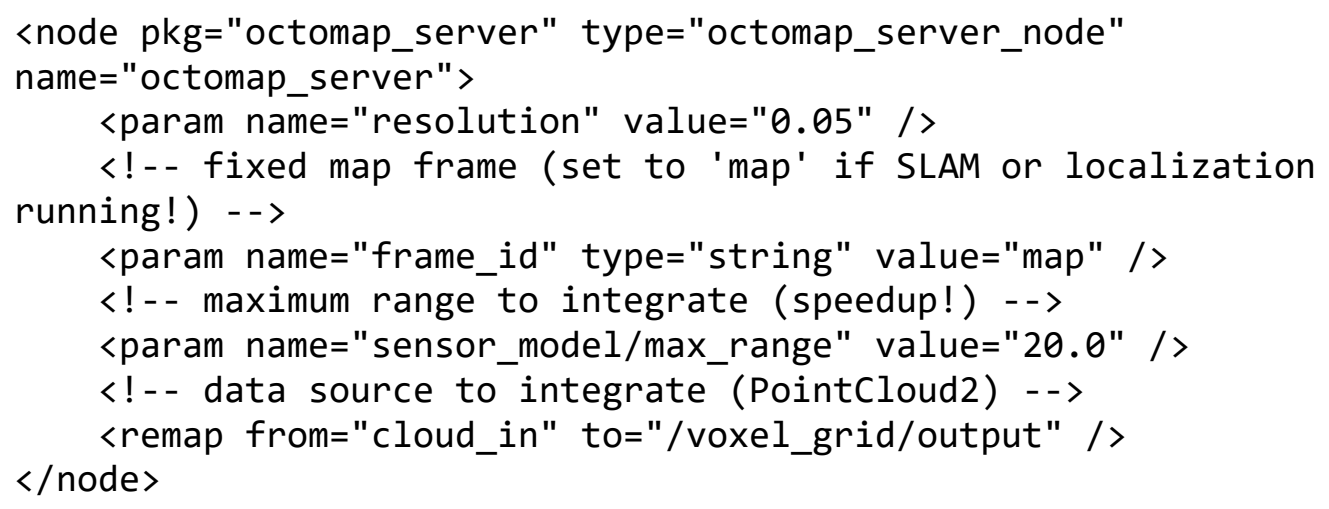


Figure 4. 3-D point cloud generated using ocotomap_server.

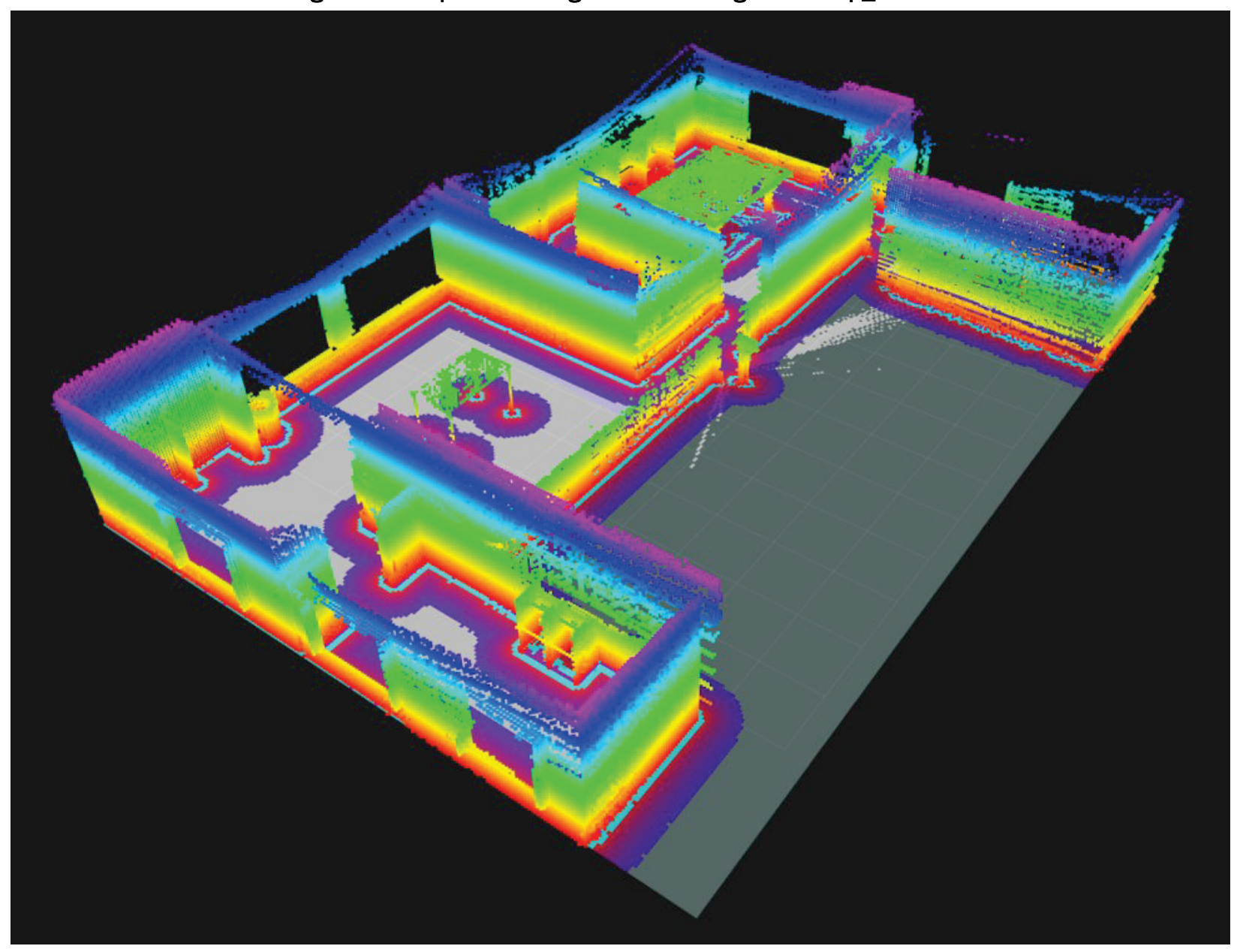

\section{RTABMap}

The third option leverages RTABMap (Labbé and Michaud 2019, 2017, 2014, 2013, and 2011). RTABMap is very capable of producing quality 3-D point clouds. Thus, the team attempted to fuse the two approaches. Running two SLAM modules at the same time is problematic, especially regarding overlapping nodes. Thus, the data_recorder node was leveraged in RTABMap. After mapping, the node will write the RTABMap database to construct a 3-D pointcloud. This approach allowed for utilization of the SLAM_toolbox to generate the occupancy grid map. Shown below is the code for the data_recorder. A number of these parameters have been discussed in previous reports.

<node name="data_recorder" pkg="rtabmap_ros" type="rtabmap" output="screen" args="--delete_db_on_start">

$<$ param name="Mem/RehearsalSimilarity" type="string" value $=1.0 " /\rangle \quad\langle$ !-- deactivate rehearsal --> 


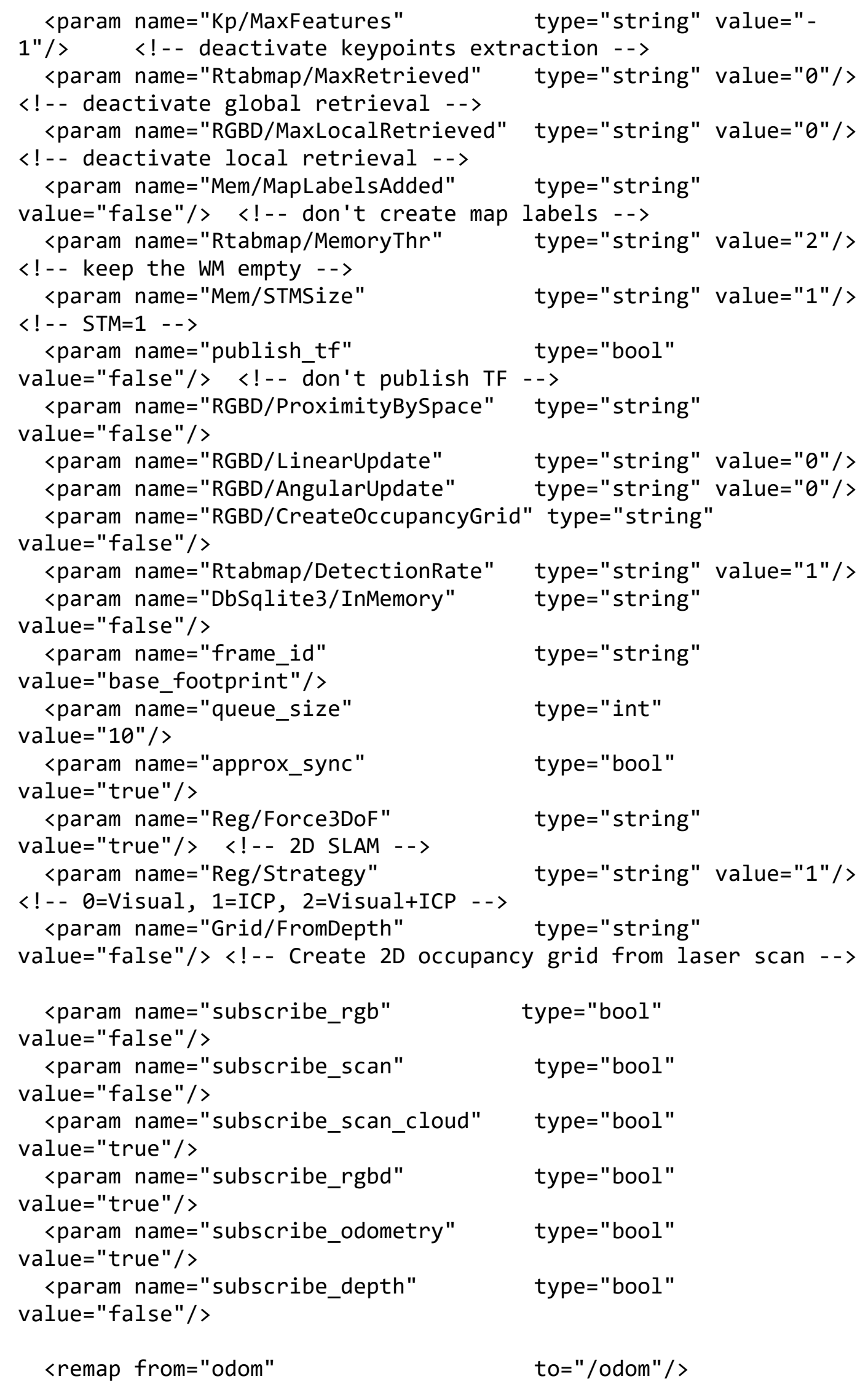




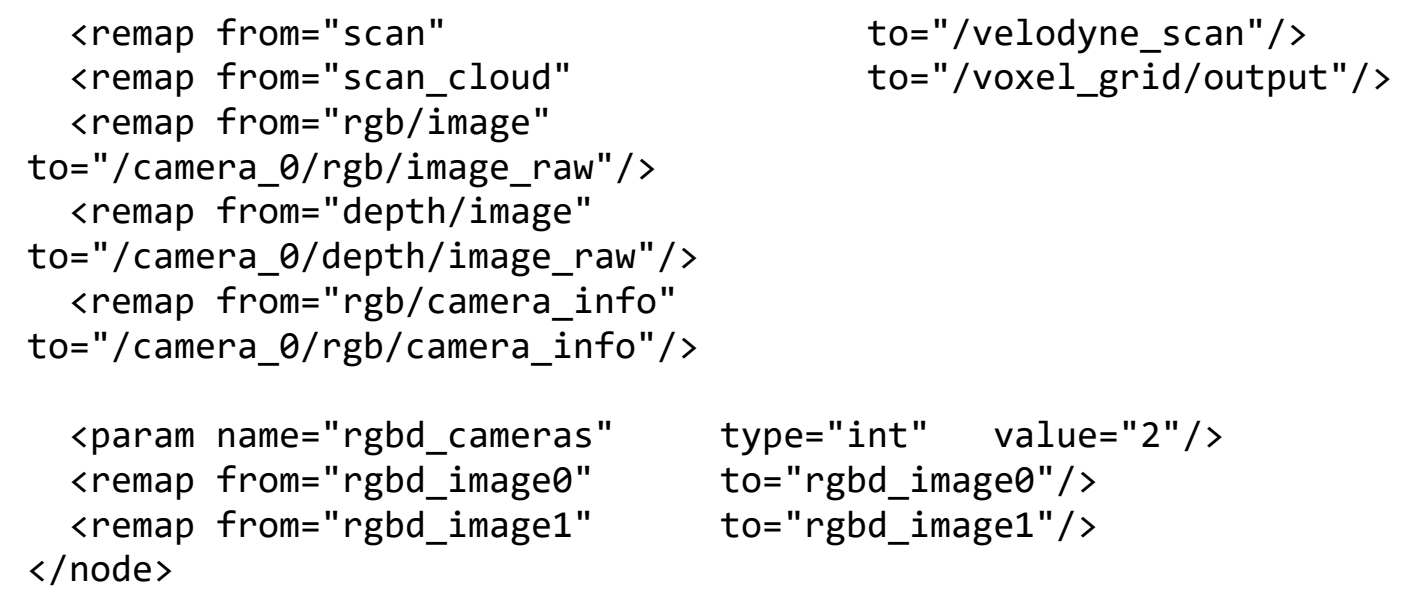

For completeness, the 2-D occupancy grid collected generated by the SLAM Toolbox is shown in Figure 5.

Figure 5. 2-D occupancy grid generated from the SLAM Toolbox when fused with RTABMap.

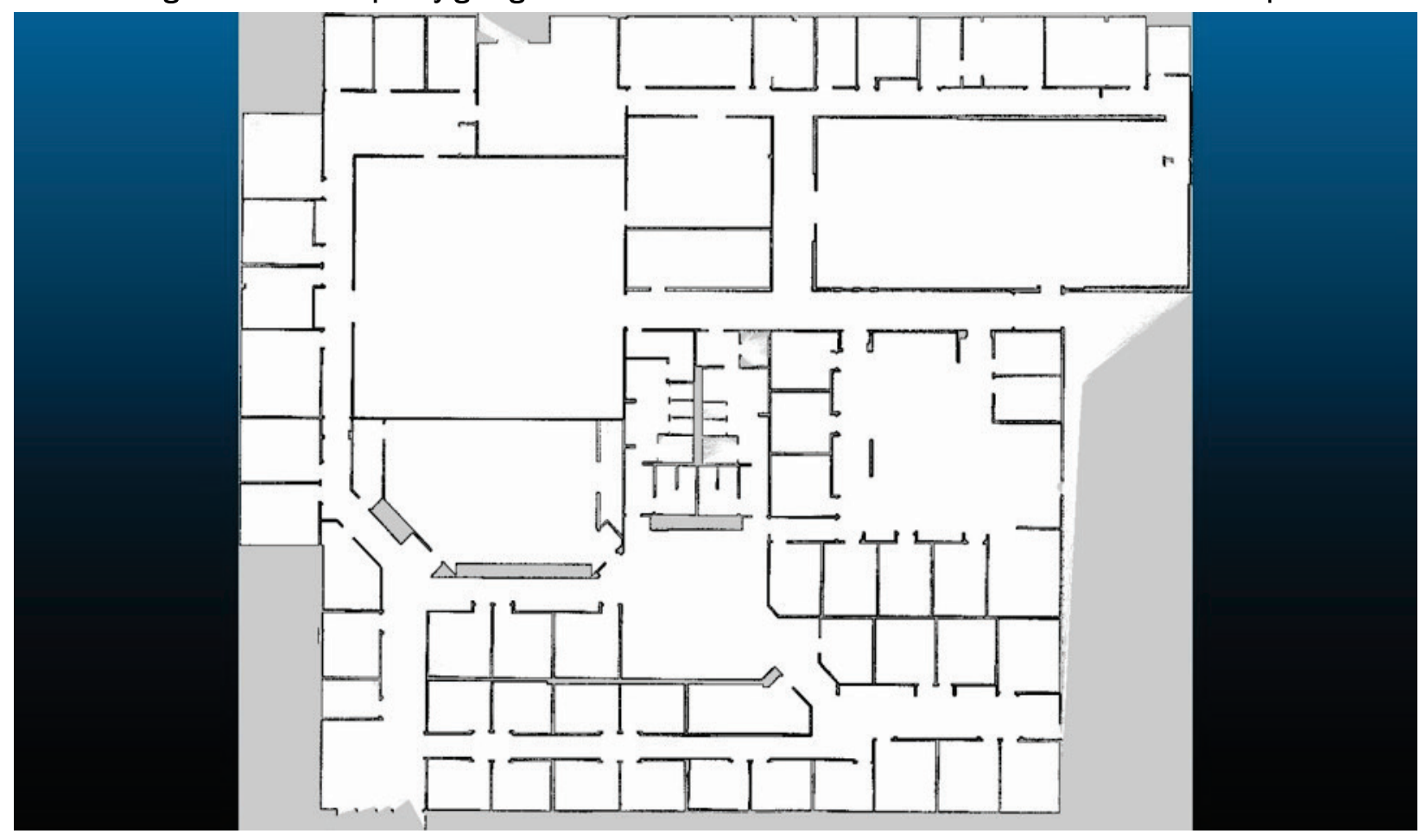

Then, RTABMap leverages the 2-D map generated by the SLAM Toobox to construct the 3-D point clouds from the collected database. Figure 6 shows the 3 -D point cloud generated from this approach. To reduce the database file size, the voxel_grid nodelet ${ }^{1}{ }^{30}$ was used to remove the ground points. In addition, adding color to the point clouds has been previously 
discussed, but is the result of combining RTABMap's

pointcloud_to_depthimage and rgbd_sync nodelets for the front and rear RGBD cameras.

Figure 6. 3-D point cloud of willow garage world generated by the SLAM Toolbox / RTABMap fusion.
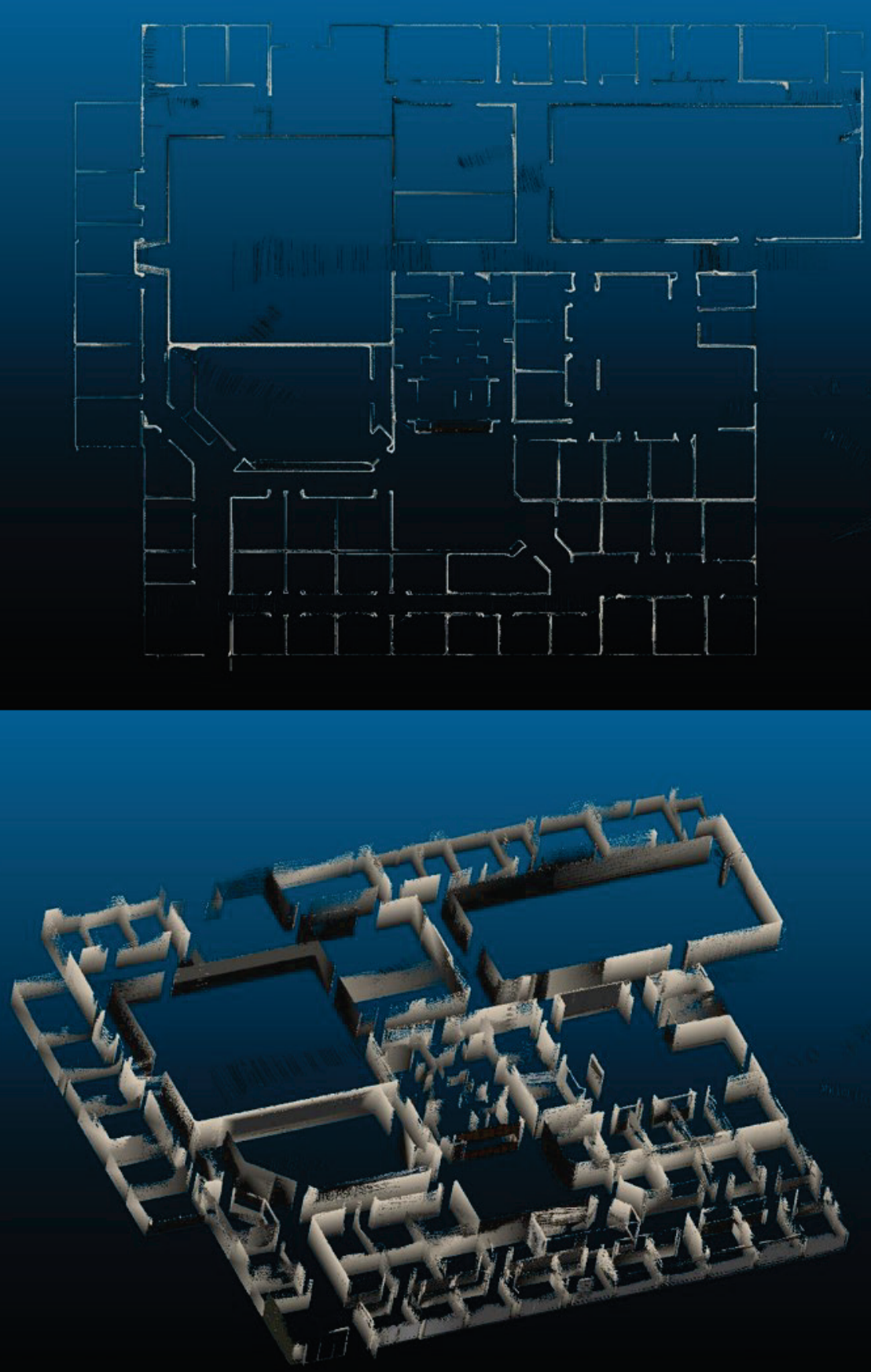
Initially, the willow garage world was chosen due to its large size. However, to highlight benefits of combining the SLAM Toolbox with RTABMap, Clearpath's playpen world was also mapped. The playpen world has significantly more visual elements compared to willow garage and highlights the benefits of this dual approach. To measure the accuracy of the generated 3-D point clouds in simulation each scan was repeated with the odometry set to ground truth. Then, the point clouds were compared in Cloudcompare and it was determined that the RMS was $\sim 3$ $\mathrm{cm}$ for both willow garage and the playpen worlds. Figure 7 shows the 3-D point cloud generated from mapping the playpen world. The full launch and configuration files can be found in Appendix B. 
Figure 7. 3-D point cloud of play pen world generated by the SLAM Toolbox / RTABMap fusion.

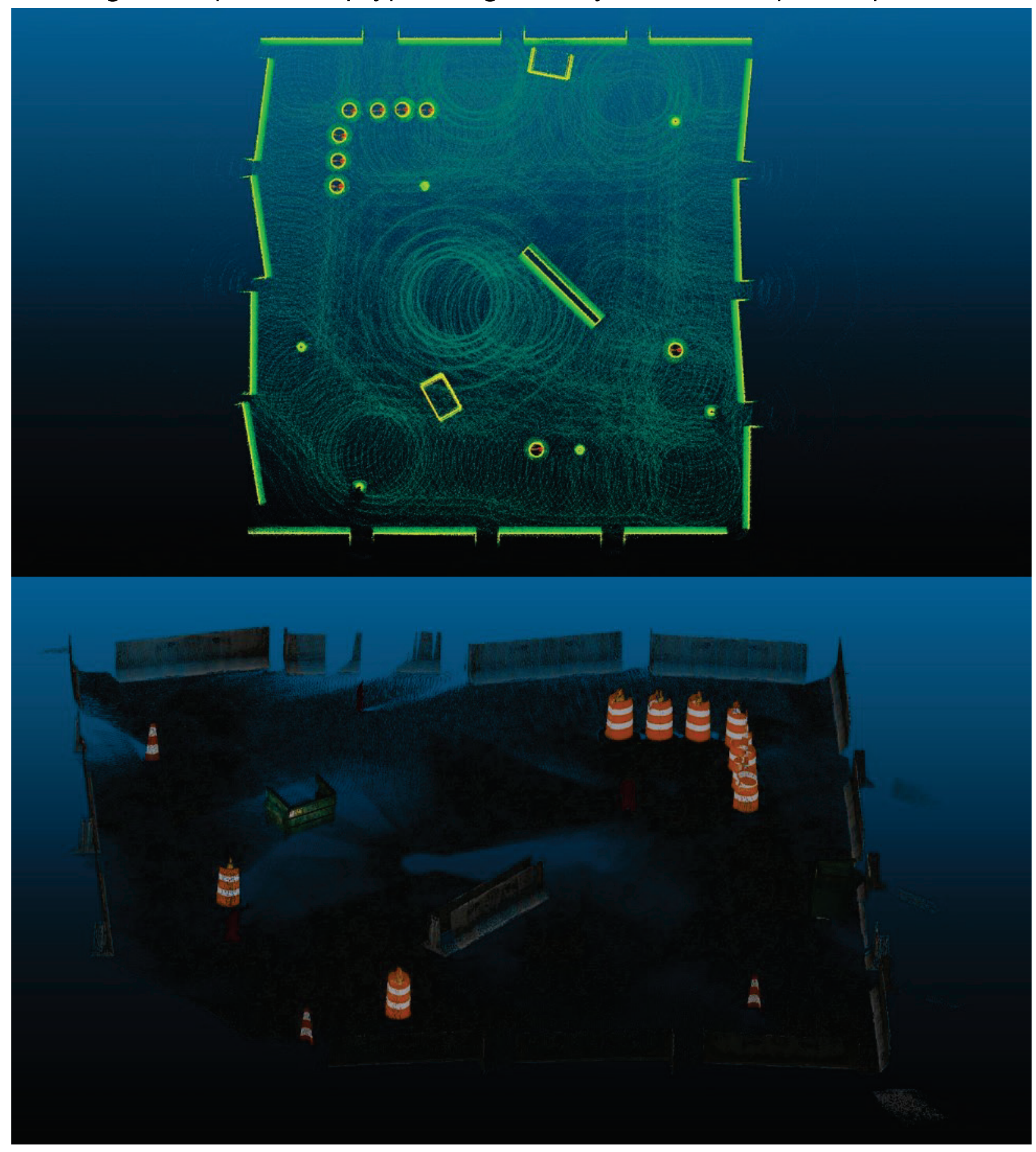




\section{Path planning using Move_base and STVL}

The purpose of the move_base package is to move the robot to its intended goal. It accomplishes this feat by utilizing global and local path planners and their respective cost maps. In this report, global_planner and eband_local_planner is used. The global_planner is quite versatile since there are choices of using $\mathrm{A} /{ }^{*}$ or Dijkstra's algorithms. When on fast moving vehicles, $\mathrm{A} /{ }^{*}$ may be preferable. Dijkstra was chosen for this study, which maximized coverage when determining the optimal path. The global_planner also possesses an orientation filter. Hypothetically, if the robot was equipped with a single camera, it would be possible to drive down a hallway and then drive backwards, using the orientation filter, on the return trip to ensure loop closures occurred in order to minimize drift. However, a dual camera approach was used; as a result, the orientation filter was not modified. Finally, the team modified the cost_factor (0.55), neutral_cost (66), and lethal_cost (253). These values are based on a report published by Kaiyuzheng. The eband_local_planner seemed to work well in this test environment. When comparing eband to the teb_local_planner out of the box, eband mapped $\sim 730 \mathrm{~m}$ in 30 min while TEB mapped $\sim 430 \mathrm{~m}$. However, development for eband seems to have stopped at kinetic in favor of teb_local_planner, which has a noetic release. However, eband did compile successfully in melodic without any issues. The global and local planners were set with the following parameters:

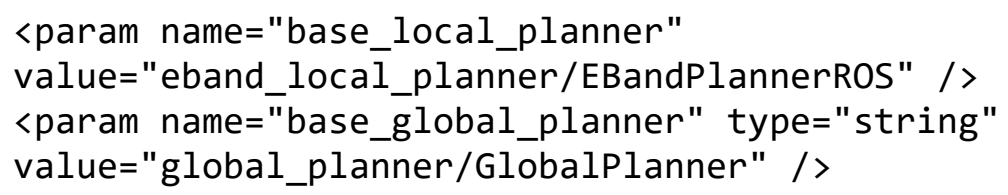

The move_base file is comprised of four sections; the global_costmap, the local_costmap, move_base and EBandPlannerROS.

Navigating in dynamic level environments is more complicated since the grid_map can change unexpectedly when objects appear, disappear, or change position within the environment. Thus, a spatio-temporal solution is necessary. The default obstacle_layer plugin generated by costmap2d can mark and clear the local_costmap using specified observation sources, such as a laser scan or pointcloud2 topic. By detecting what surfaces are obstacles and what is free space, clearing can occur within the costmap. Thus, if an obstacle moves, the space that the obstacle occupied is now 
free, and cleared. This was accomplished by using the spatio_temporal_voxel_layer::rgbd_obstacle_layer (STVL) plugin. The STVL is a direct drop-in replacement for the costmap2d::voxel_layer plugin for collision avoidance, path planning, and control ${ }^{1}$. In dynamic environments, STVL can leverage depth cameras or a 3-D LiDAR. In this report, the pointcloud from the 3-D LIDAR was chosen for marking and clearing and shown in Figure 8. The complete launch file for move_base including STVL can be found in Appendix C.

Figure 8. Costmap, with inflation layer, generated using the spatio_temporal_voxel_layer.

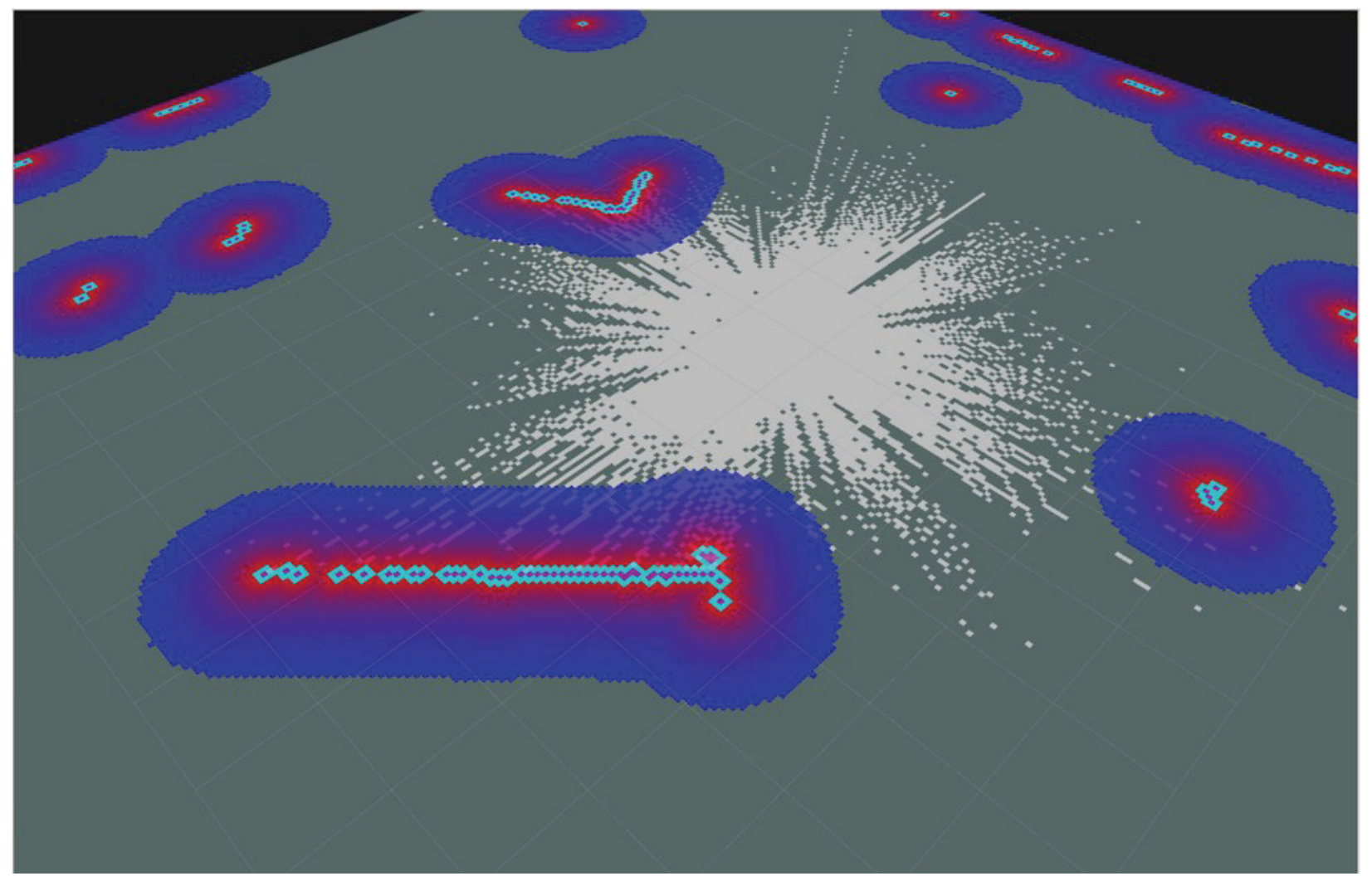

1 https://github.com/SteveMacenski/spatio_temporal_voxel_layer 


\section{Autonomous Frontier-based Exploration using Explore Lite}

In this report, explore_lite was leveraged to send goals to the path planner. However, explore_lite does not have the same virtual fencing capabilities that exists in frontier_exploration, another popular frontierbased exploration application. Ultimately, this means that in unbounded environments, explore_lite will endlessly send new navigation goals to the path-planning software. The cycle repeats if there are new frontiers to navigate. This can be seen in Figure 9. Consequently, a virtual fence was added to explore_lite. This was accomplished by modifying FrontierSearch::isNewFrontierCell to remove frontiers whose pose did not meet a specified positional requirement. As a proof-of-concept, maximum $\mathrm{x}$ and $\mathrm{y}$ values were used to create a square virtual fence in simulation. Explore_lite explored the square in an open, unbounded environment, and explore_lite did not endlessly explore. Furthermore, Explore::stop was modified to send a navigation goal to the path-planner once exploration was finished so the robot could return to its original position, or a specified ending position. The files modified for virtual fencing and return to home are found in Appendix D. 
Figure 9. Frontier-based exploration using explore_lite over time. The frontiers are blue, and their associated costs are depicted by the green circles. The red dots depict the path of the robot and obstacles are marked with an inflation layer.

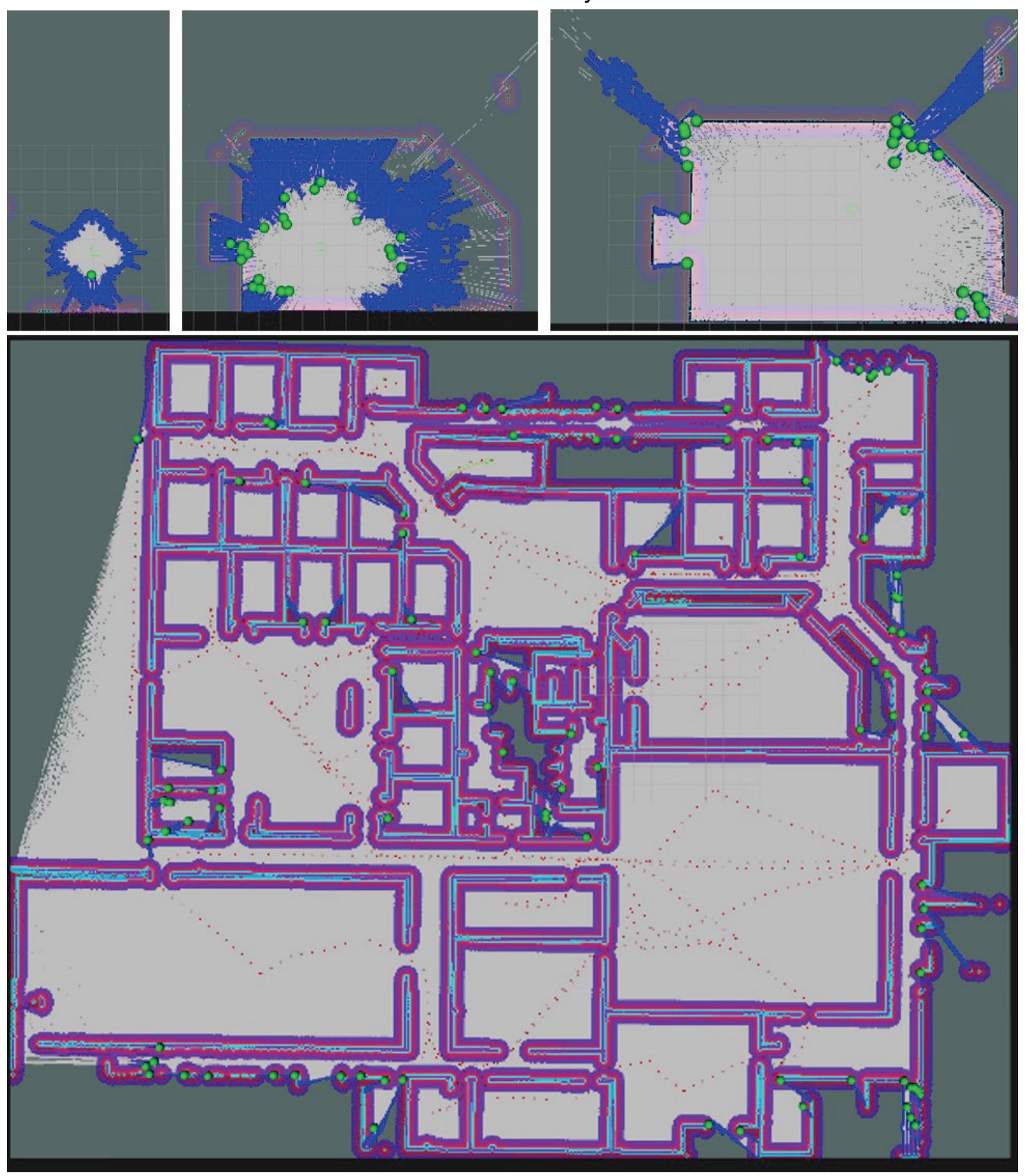




\section{Conclusion}

Finally, autonomy exploration and navigating locally in a 2-D environment is achievable by implementing all the previously discussed packages. This report has demonstrated that 3-D RGB point clouds can be generated in simulation mapping $>900 \mathrm{~m}$ with an RMS of $\sim 3 \mathrm{~cm}$. To increase the robustness of this approach for a real-world application, pointcloud_to_laserscan was leveraged for obstacle detection and STVL for environments with dynamic obstacles. Eventually, moving towards a voxel-based implementation for automating 3 -D environments may be in the team's best interest. In this approach, maps are projected to a twodimensional occupancy grid for navigation. However, if a tunnel spirals back on itself such that a point $A(X, Y, a)$ exists within the map and also a point $B(X, Y, b)$ exists within the map such that $\mathrm{A}$ and $\mathrm{B}$ are vertically coincident while $a \neq b$, the terrain immediately surrounding A and B will become superimposed within the global_costmap, and navigation will likely fail. However, octomaps use a three-dimensional approach instead, with voxels instead of two-dimensional cells. Consequently, unique points with the same $\mathrm{X}$ and $\mathrm{Y}$ values, but unique $\mathrm{Z}$ values, can exist and affect navigation separately. Unfortunately, not many implementations of this idea exist. One package that has this idea is MoveIt. Future work will focus on leveraging this type of package to enhance global navigation. 


\section{References}

Castellanos, J. A., J. Neira, and J. D. Tardós.2001. "Multisensor fusion for simultaneous localization and map building." IEEE Transactions on Robotics and Automation, 17.6: 908-914.

Ekvall, S., P. Jensfelt, and D. Kragic. 2006. "Integrating active mobile robot object recognition and slam in natural environments." IEEE/RSJ International Conference on Intelligent Robots and Systems.

Glaspell, Garry. P., S. R. Lessard, B. A. Christie, K. Jannak-Huang, N. C. Wilde, W. He, O. Ennasr, D. T. Pham, D. B. Hasemann, P. M. Devine, and J. Kiene. 2020. Optimized low Size, Weight, Power and Cost (SWaP-C) payload for mapping interiors and subterranean on an Unmanned Ground Vehicle. ERDC/GRL TR20-6. Alexandria, VA: U.S. Army Engineer Research and Development Center.

Guanci, Y., Z. Chen, Y. Li, and Z. Su. 2019. "Rapid Relocation Method for Mobile Robot Based on Improved ORB-SLAM2 Algorithm." Remote Sensing, 11.2: 149.

Jacobson, A., F. Zeng, D. Smith, N. Boswell, T. Peynot, and M. Milford. 2018. "Semisupervised slam: Leveraging low-cost sensors on underground autonomous vehicles for position tracking." IEEE/RSJ International Conference on Intelligent Robots and Systems (IROS).

Keidar, M., and G. A. Kaminka. 2014. "Efficient frontier detection for robot exploration." The International Journal of Robotics Research, 33(2):215-236. doi:10.1177/0278364913494911

Labbé, M., and F. Michaud. 2019. "RTAB-Map as an Open-Source Lidar and Visual SLAM Library for Large-Scale and Long-Term Online Operation." Journal of Field Robotics, 36(2): 416-446.

Labbé, M., and F. Michaud. 2017. "Long-term online multi-session graph-based SPLAM with memory management." Autonomous Robots, 42(6): 1133-1150.

Labbé, M., and F. Michaud. 2014. "Online Global Loop Closure Detection for Large-Scale Multi-Session Graph-Based SLAM.” In Proceedings of the IEEE/RSJ International Conference on Intelligent Robots and Systems.

Labbé, M., and F. Michaud. 2013. "Appearance-based loop closure detection in real-time for large-scale and long-term operation.” IEEE Transactions on Robotics, 29(3): 734-745.

Labbé, M., and F. Michaud. 2011. "Memory management for real-time appearance-based loop closure detection.” In Proceedings of the IEEE/RSJ International Conference on Intelligent Robots and Systems.

Roman, L., A. Zakiev, E. Magid, M. Svinin, and F. Matsuno. 2019. "Partially Unknown Environment Exploration Algorithm for a Mobile Robot." Journal of Advanced Research in Dynamical and Control Systems, 11: 1743-1753. 
Wang, D., H. Wang, L. Liu. 2016. "Unknown environment exploration of multi-robot system with the FORDPSO, Swarm and Evolutionary Computation." Volume 26 157-174, ISSN 2210-6502.

Wang, Y., A. Liang, and H. Guan. 2011. "Frontier-based multi-robot map exploration using Particle Swarm Optimization," IEEE Symposium on Swarm Intelligence, Paris, pp. 1-6, doi: 10.1109/SIS.2011.5952584. 


\section{Appendix A}

\section{turtlebot3_waffle_3d2.gazebo.xarco}

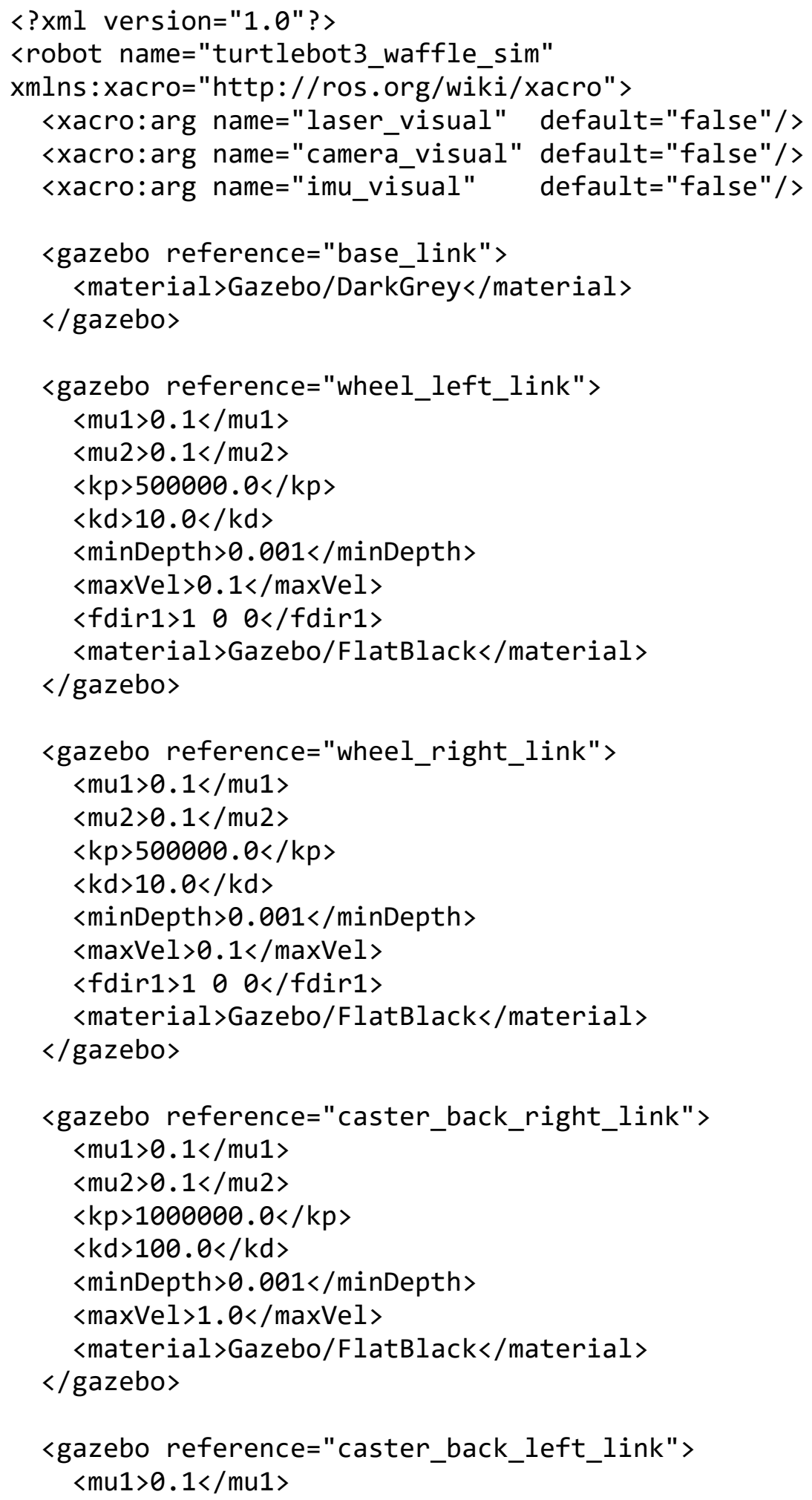




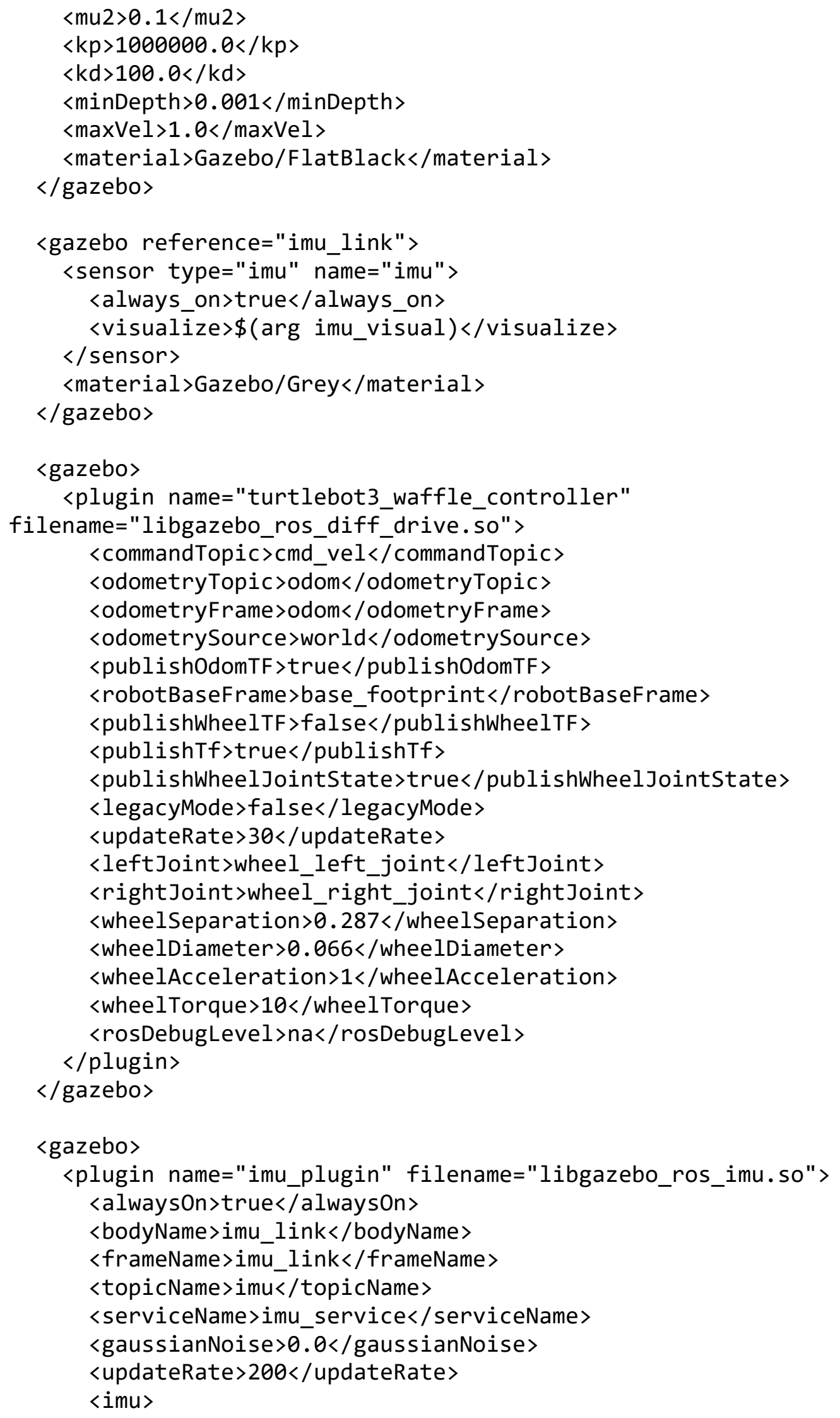




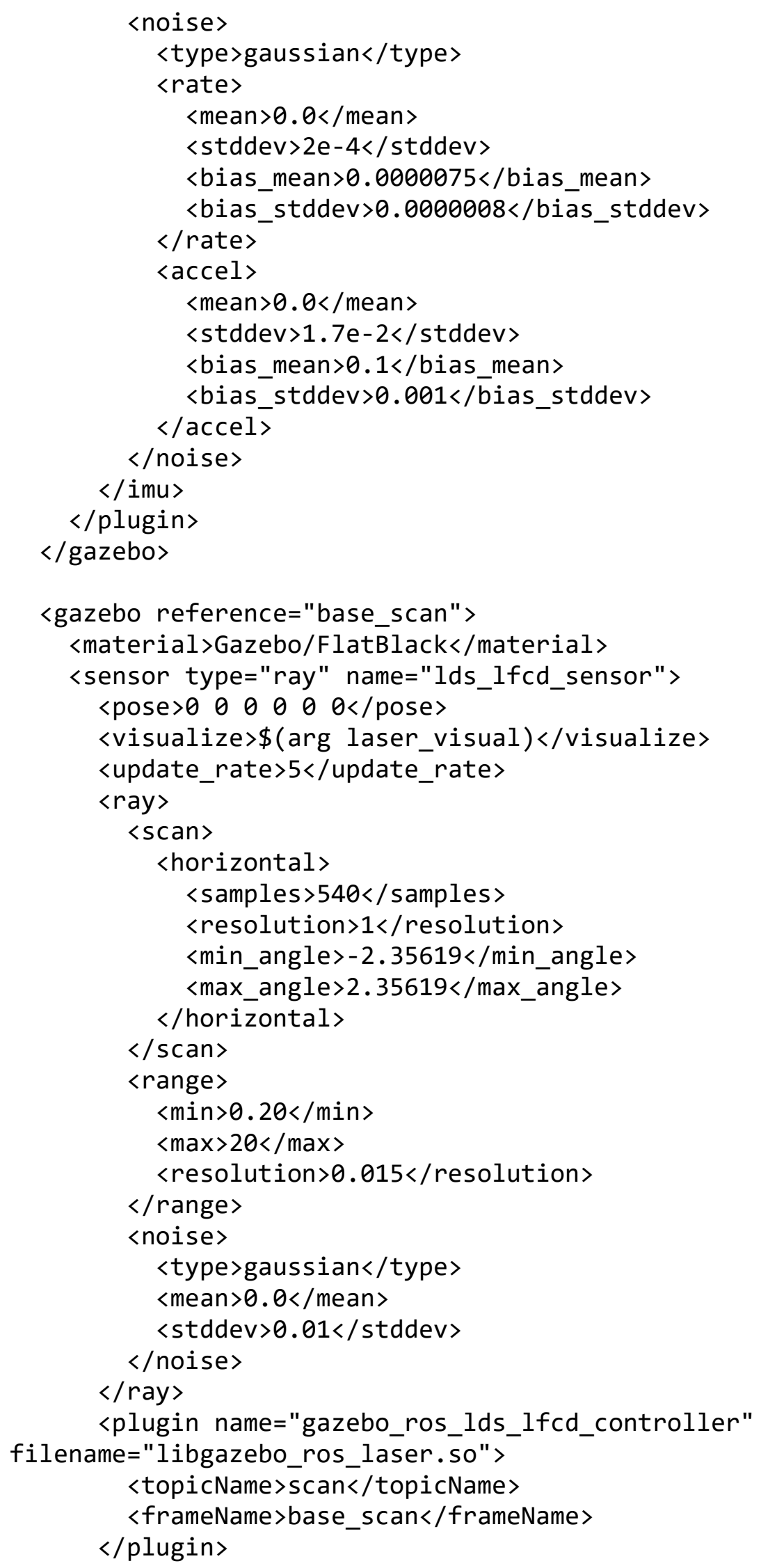




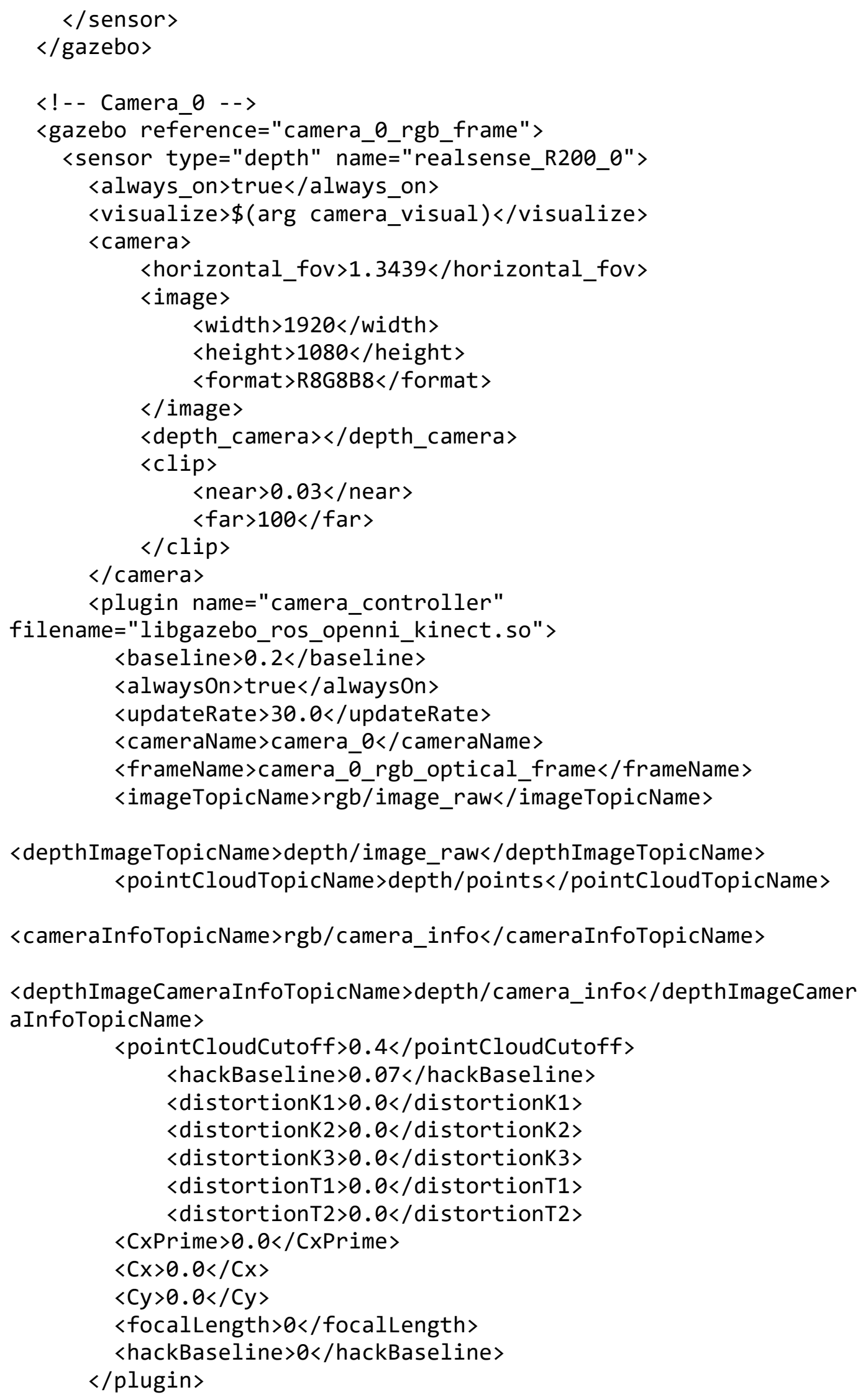




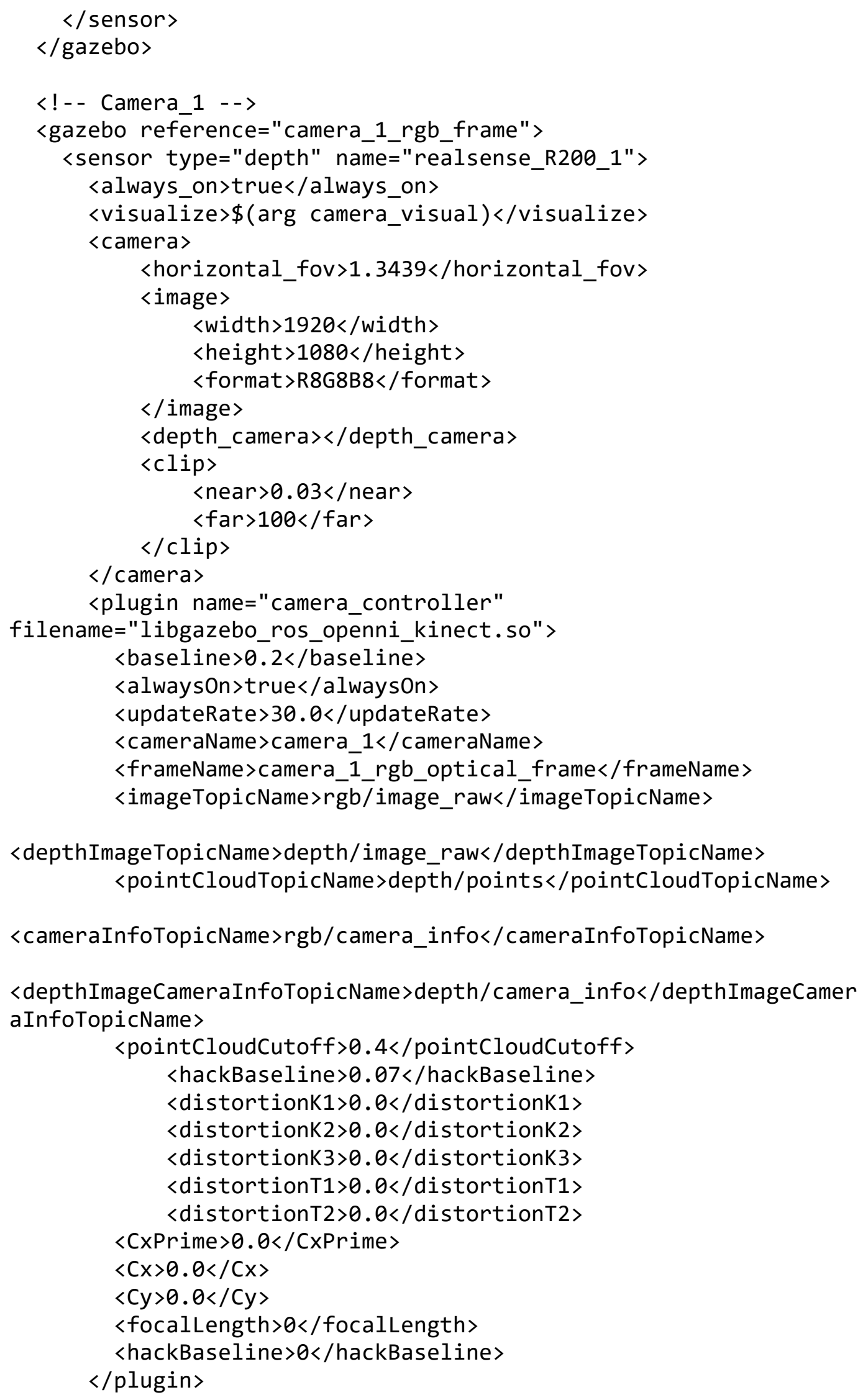




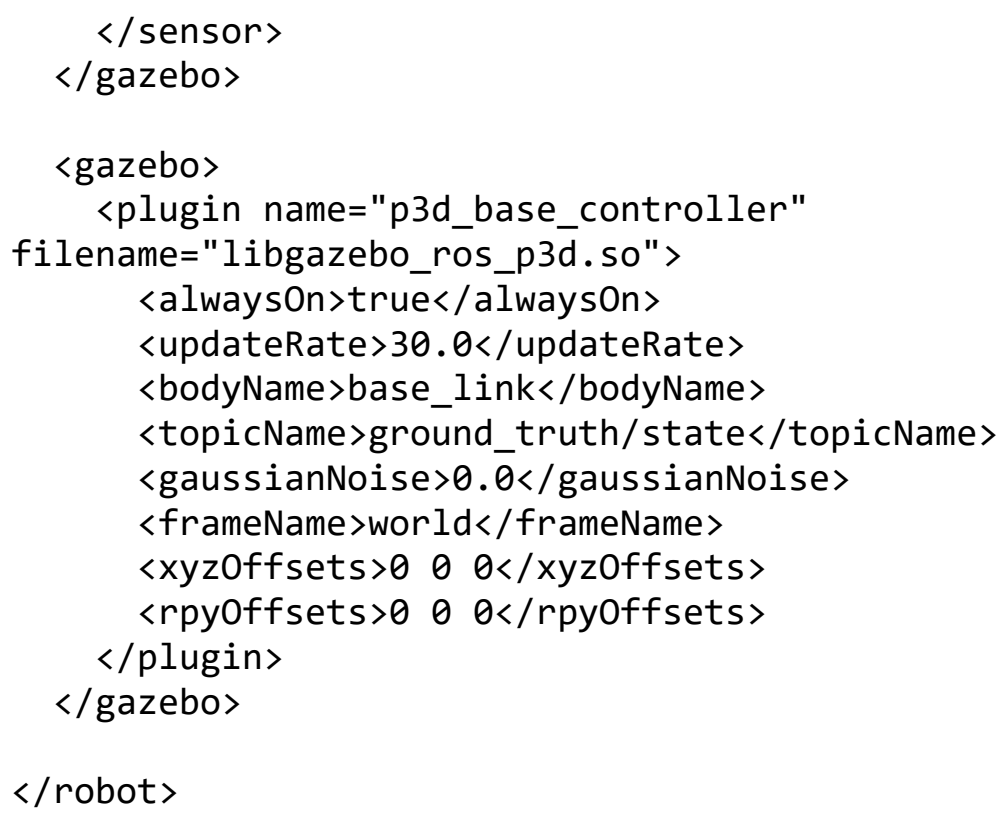

\section{turtlebot3_waffle_3d_2.urdf.xarco}

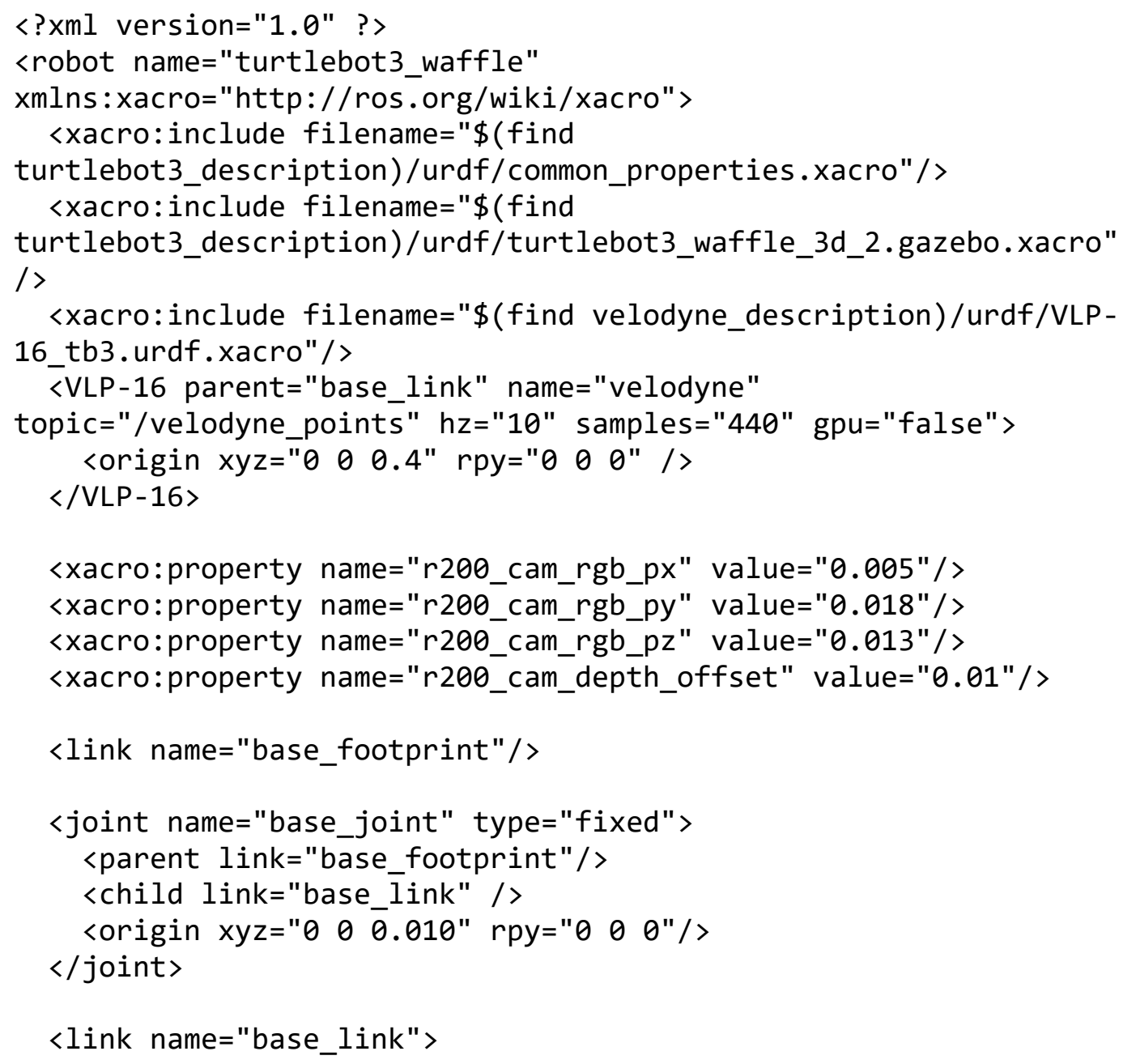




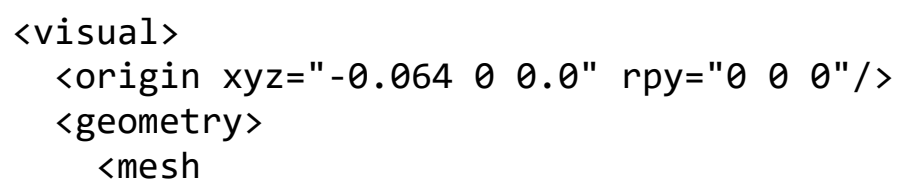

filename="package://turtlebot3_description/meshes/bases/waffle_ba se.stl" scale="0.001 $0.0010 .001 " />$

$\langle/$ geometry>

$\langle$ material name="light_black"/>

$\langle/$ visual $>$

$\langle$ collision>

<origin $x y z="-0.06400 .047 "$ rpy="0 $000 " />$

<geometry>

<box size="0.266 $0.2660 .094 " />$

$\langle/$ geometry $>$

$\langle/$ collision $>$

<inertial>

<origin $x y z=" \begin{array}{llllll}0 & 0 & 0 " & \text { rpy }=" 0 & 0 & 0 " />\end{array}$

<mass value $=" 1.3729096 \mathrm{e}+00 " />$

<inertia ixx="8.7002718e-03" ixy="-4.7576583e-05"

ixz="1.1160499e-04"

iyy $=" 8.6195418 e-03 "$ iyz="-3.5422299e-06"

$\langle/$ inertial $\rangle$

izz="1.4612727e-02" />

$\langle/$ link $>$

〈joint name="wheel_left_joint" type="continuous"〉

<parent link="base_link"/>

<child link="wheel_left_link"/>

<origin $x y z=" 0.00 .144 \overline{0} .023 "$ rpy $="-1.5700 "$ "/>

<axis $x y z=" 0 \quad 0 \quad 1 " />$

$\langle/$ joint $\rangle$

〈link name="wheel_left_link" >

$\langle$ visual>

<origin $x y z=" 0 \quad 0 \quad 0 "$ rpy=" $\begin{array}{llllll}1.57 & 0 & 0 & 0 & 0\end{array}$

<geometry>

$<$ mesh

filename="package://turtlebot3_description/meshes/wheels/left_tir

e.stl" scale="0.001 $0.0010 .001 " />$

$\langle/$ geometry>

$<$ material name="dark" / >

$\langle/$ visual $>$

$<$ collision>

<origin $x y z=" \begin{array}{lllllll}0 & 0 & 0 & \text { rpy="0 } & 0 & 0 & 0\end{array}$

<geometry>

<cylinder length $=0.018 "$ radius $=" 0.033 " />$

$</$ geometry $>$ 


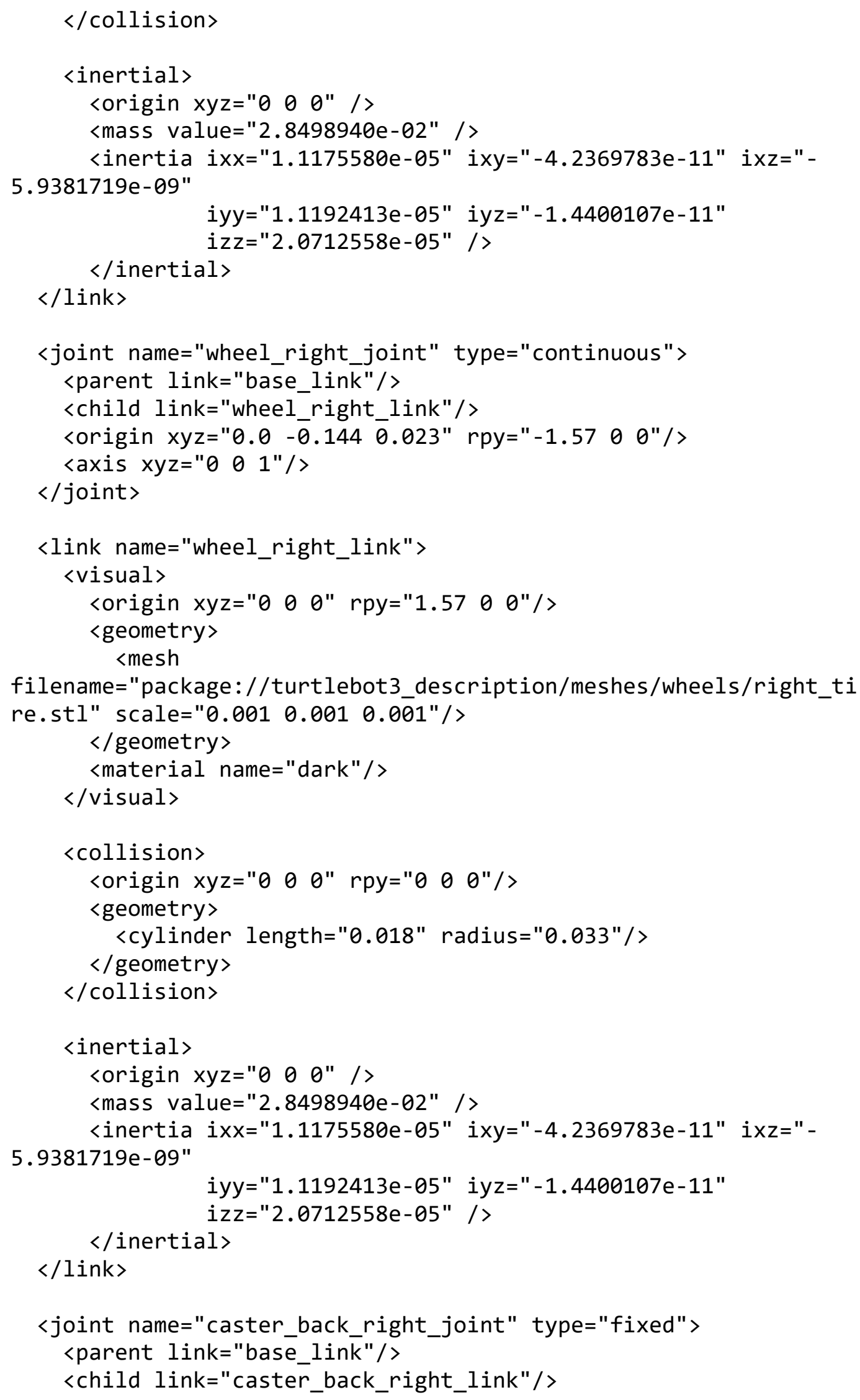




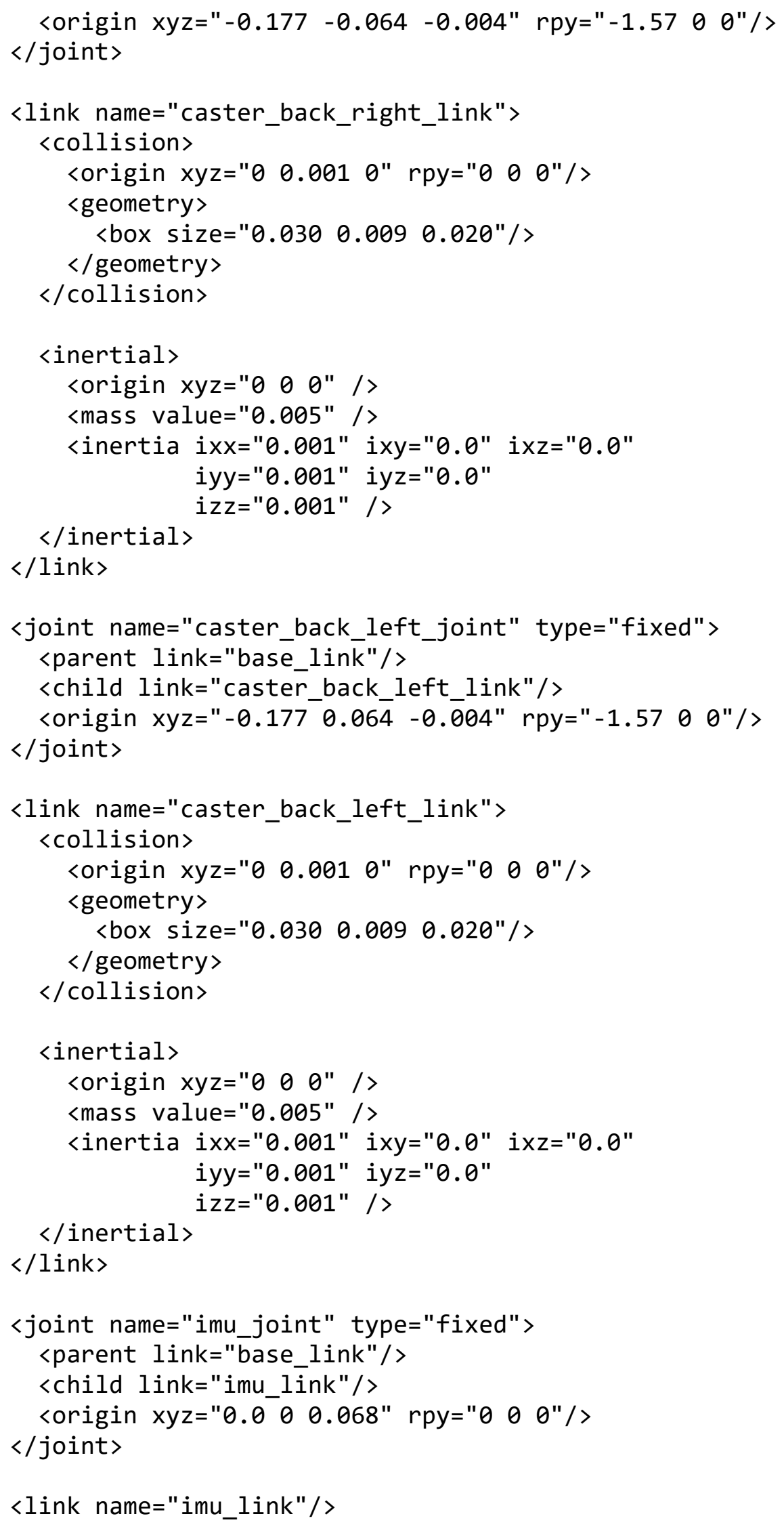




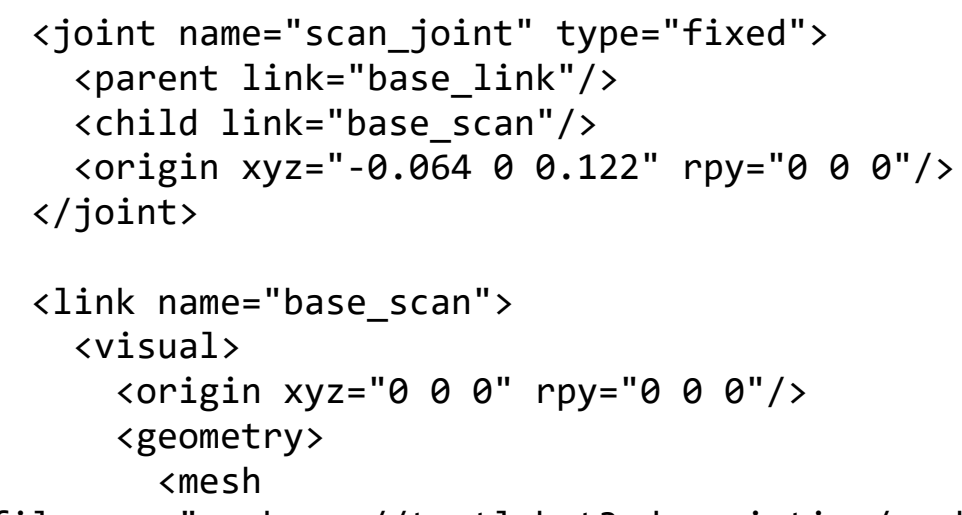

filename="package://turtlebot3_description/meshes/sensors/lds.stl

" scale="0.001 $0.0010 .001 " />$

$\langle/$ geometry> $<$ material name="dark" / >

$\langle/$ visual $>$

$<$ collision>

<origin xyz="0.015 0 - $0.0065 "$ rpy="0 00 0"/>

<geometry>

$\langle$ cylinder length $=" 0.0315 "$ radius $=" 0.055 " />$

$\langle/$ geometry $\rangle$

$</$ collision $>$

<inertial>

<mass value $=" 0.114 " />$

<origin $x y z=" \begin{array}{llll}0 & 0 & 0 " />\end{array}$

<inertia ixx="0.001" ixy="0.0" ixz="0.0"

iyy $=" 0.001 "$ iyz="0.0"

izz $=" 0.001 " />$

$\langle/$ inertial $\rangle$

$\langle/$ link $>$

$\langle$ !-- Camera_o -->

$\langle$ joint name="camera_o_joint" type="fixed" >

<origin xyz="0.064 -0.065 $0.094 "$ rpy="0 00 0"/>

<parent link="base_link"/>

<child link="camera_o_link"/>

$\langle/$ joint $\rangle$

$\langle$ link name="camera_0_link" >

$\langle$ visual>

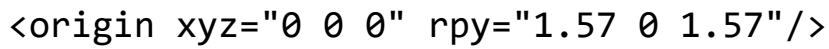

<geometry>

$<$ mesh

filename="package://turtlebot3_description/meshes/sensors/r200.da e" />

$</$ geometry>

$\langle/$ visual $>$ 


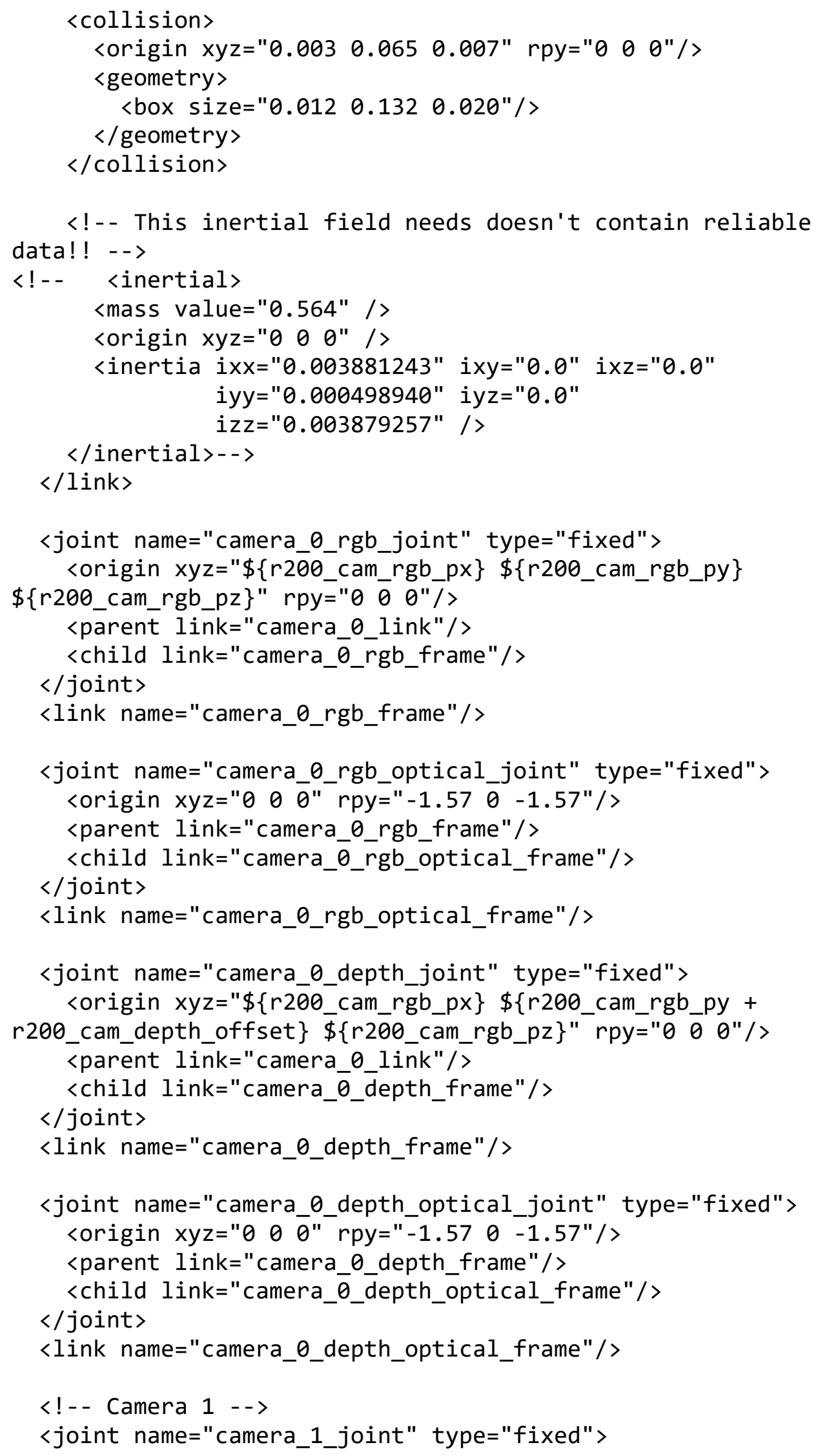




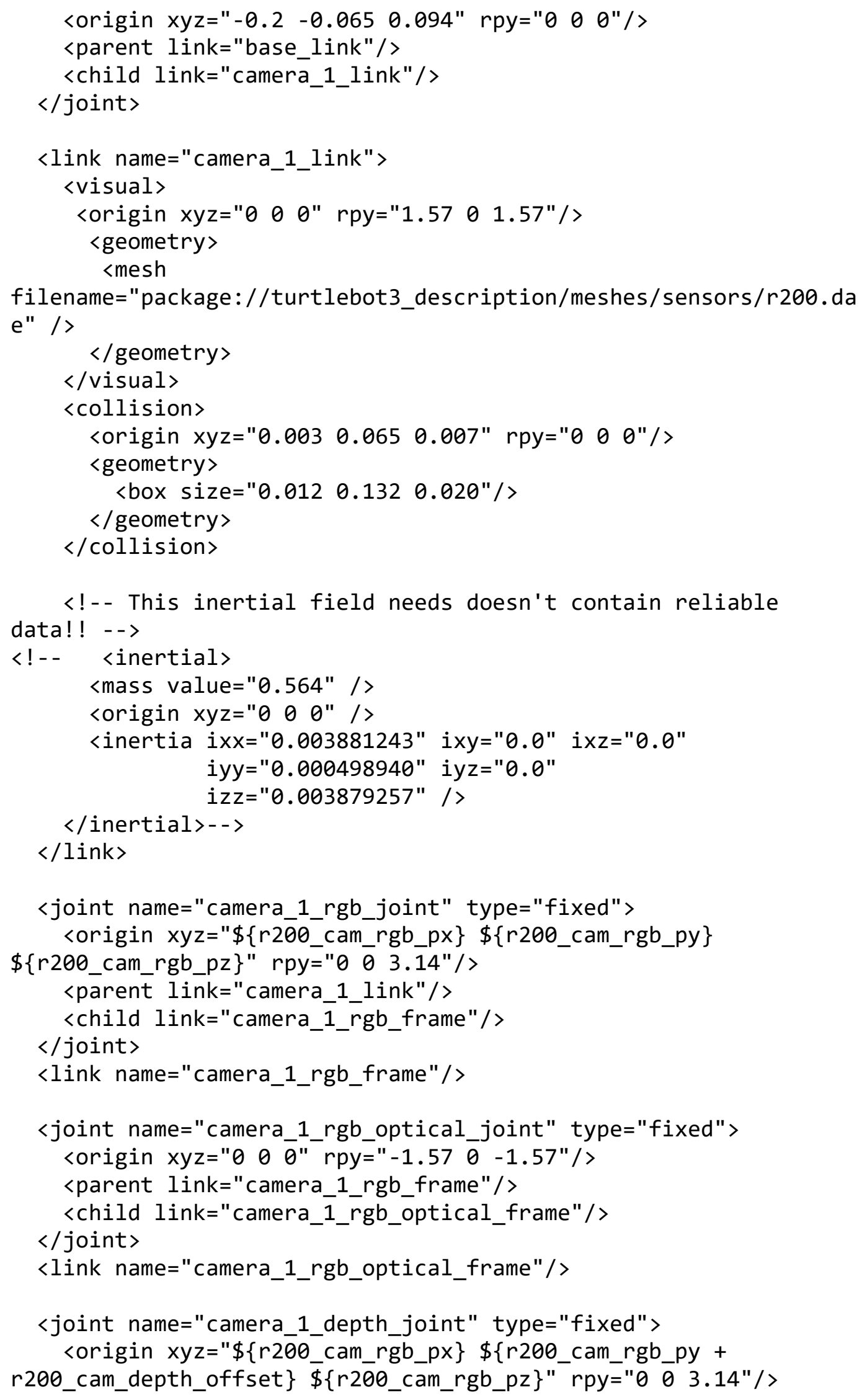




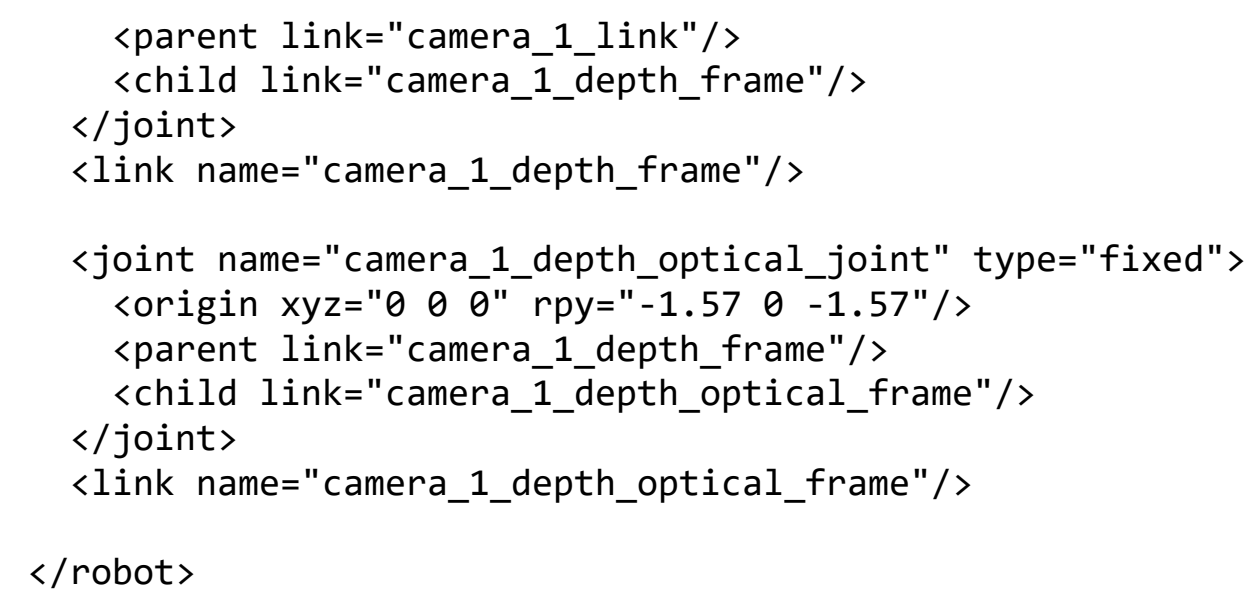

\section{turtlebot3_willow_3d_2.launch}

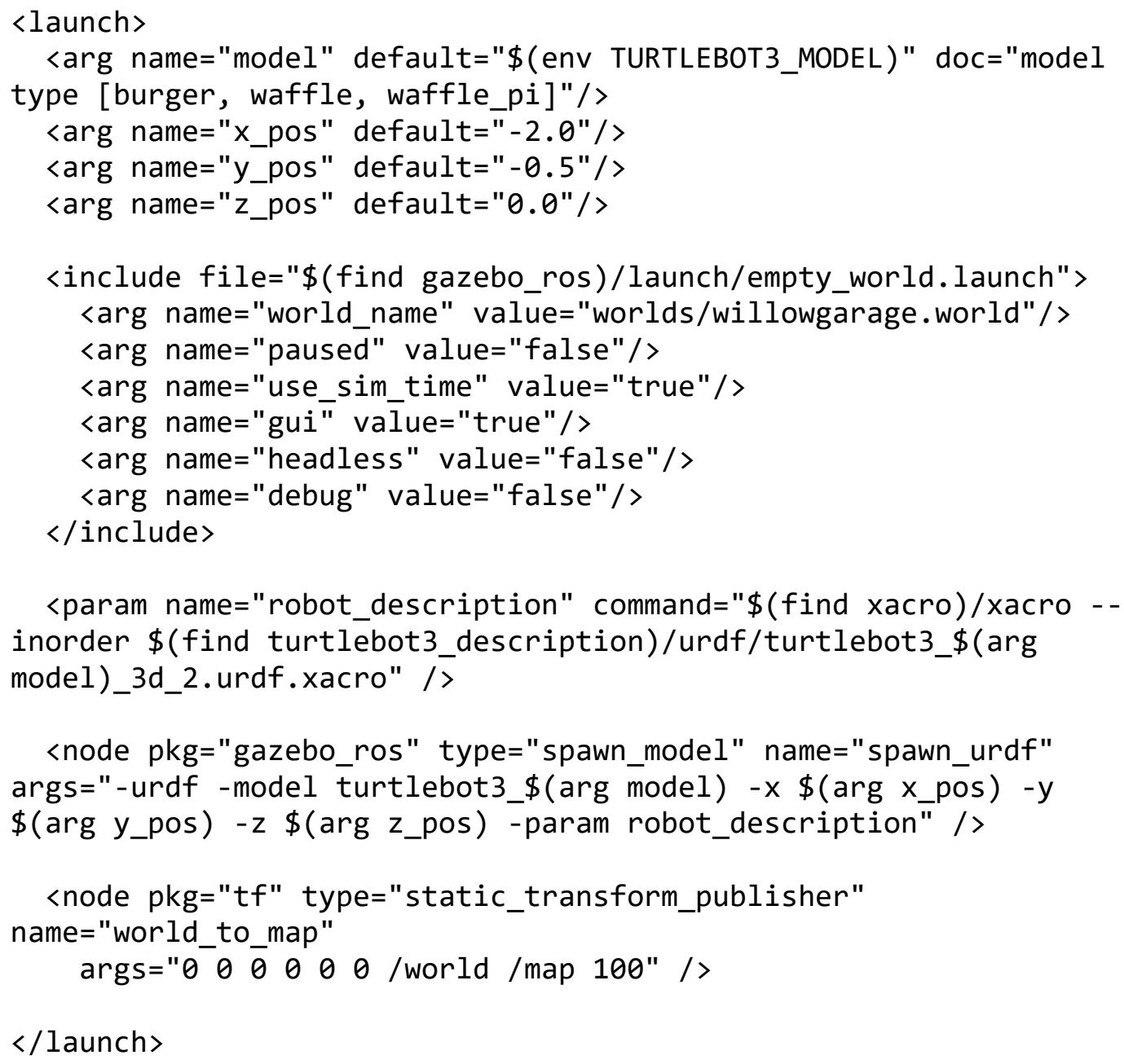




\section{Appendix B}

\section{tb3_online_sync_rtab.launch}

$\langle$ launch>

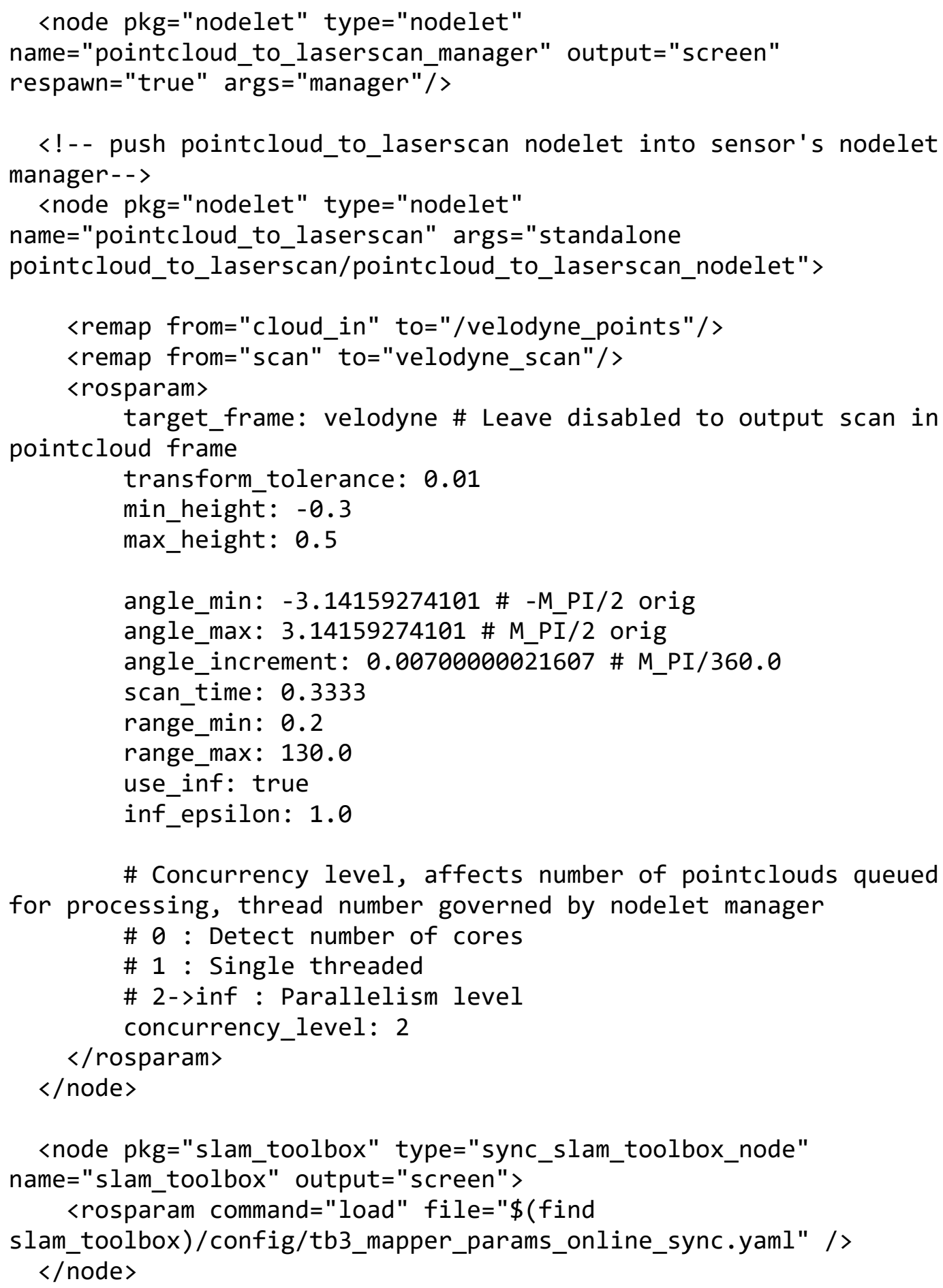


<node pkg="tf" type="static_transform_publisher" name="foot_to_scan" args $="-0.06400 .122000$ /base_footprint /base_scan 100" />

<node pkg="tf" type="static_transform_publisher" name $="$ foot_to_velodyne" args $=" \begin{array}{lllllll}0 & 0 & 0.44 & 0 & 0 & 0 & \text { /base_footprint }\end{array}$ /velodyne $100 "$ />

<node pkg="tf" type="static_transform_publisher" name="foot_to_rgb_0" args="0.064 $-0.065 \quad 0.094-1.57 \quad 0 \quad-1.57$

/base_footprint /camera_0_rgb_optical_frame 100" /> <node pkg="tf" type="static_transform_publisher" name="foot_to_depth_0" args="0.064 $-0.0650 .094-1.57 \quad 0 \quad-1.57$ /base_footprint /camera_o_depth_optical_frame 100" /> <node pkg="tf" type="static_transform_publisher" name="foot_to_rgb_1" args="-0.2 $-0.0650 .0941 .57 \quad 0 \quad-1.57$ /base_footprint /camera_1_rgb_optical_frame 100" /> <node pkg="tf" type="static_transform_publisher" name="foot_to_depth_1" args="-0.2 $\quad-0.0650 .094 \quad 1.57 \quad 0 \quad-1.57$ /base_footprint /camera_1_depth_optical_frame 100" />

<node pkg="nodelet" type="nodelet" name="pcl_manager" args="manager" output="screen" / > <node pkg="nodelet" type="nodelet" name="voxel_grid" args="load pcl/VoxelGrid pcl_manager" output="screen"> $\langle$ remap from=" input" to="/velodyne_points" / > $\langle$ rosparam>

filter_field_name: $z$

filter_limit_min: -0.4 \# -1000 is abs min

filter limit max: 10 \# 1000 is abs max

filter_limit_negative: False

leaf_size: 0.01

$</$ rosparam $>$

$\langle/$ node $>$

<node pkg="nodelet" type="nodelet"

name="pointcloud_to_depthimage_ $\theta$ " args="standalone

rtabmap_ros/pointcloud_to_depthimage" output="screen"> $<$ remap from="camera_info"

to=" / camera_o/rgb/camera_info"/> <remap from="cloud" to="/voxel_grid/output"/> $\langle$ remap from="depth_topic" to="image_raw"/> <remap from="image_raw" to="image_raw_0" / > $<$ param name="fixed_frame_id" type="string"

value="velodyne" / > $\langle$ param name="fill_holes_size" type="int" value="30"/> <param name="decimation" type="int" value="1"/> $\langle/$ node $>$

<node pkg="nodelet" type="nodelet" name="rgbd_sync_o" args="standalone rtabmap_ros/rgbd_sync" output="screen"> <remap from="rgb/image" 


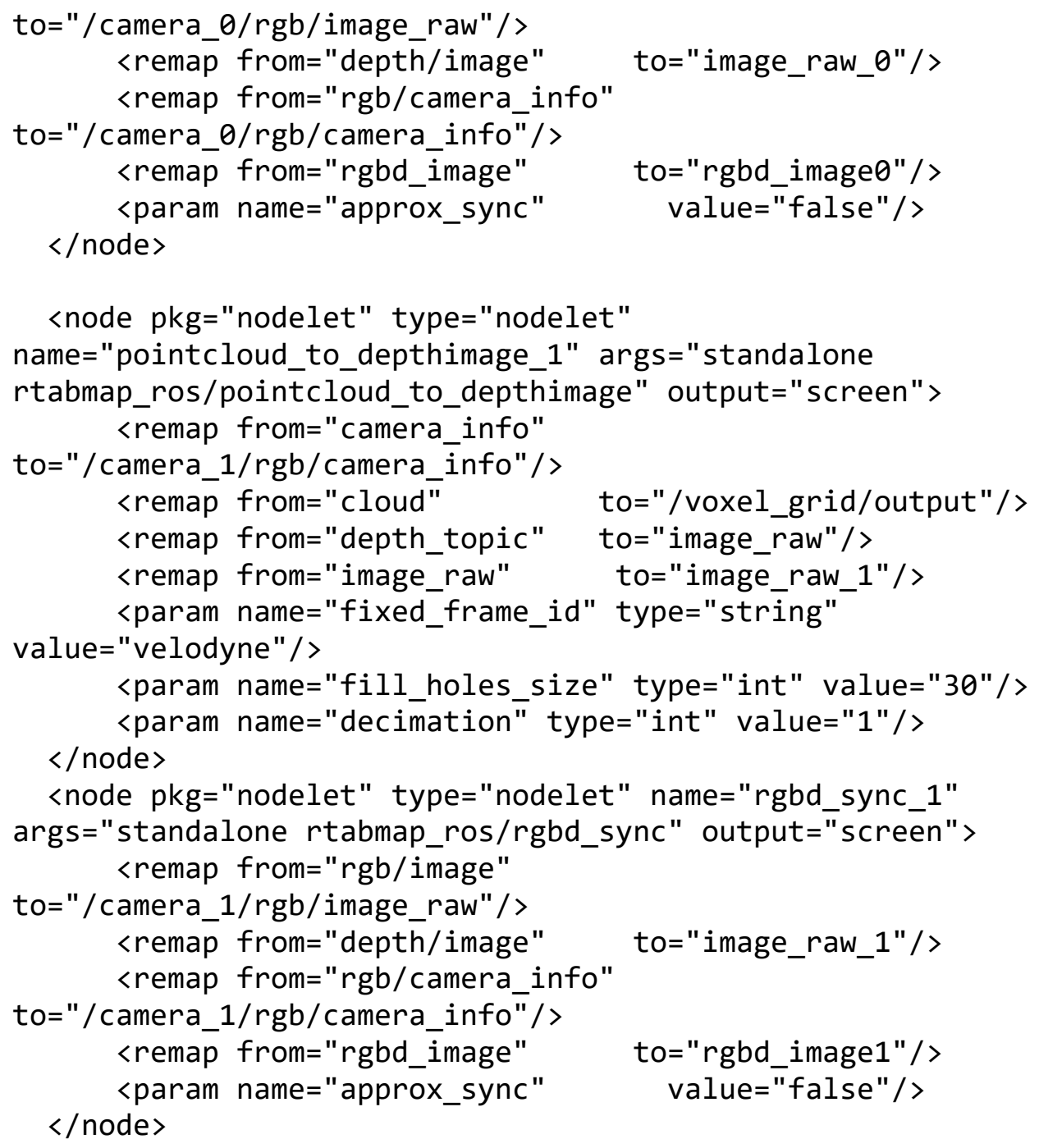




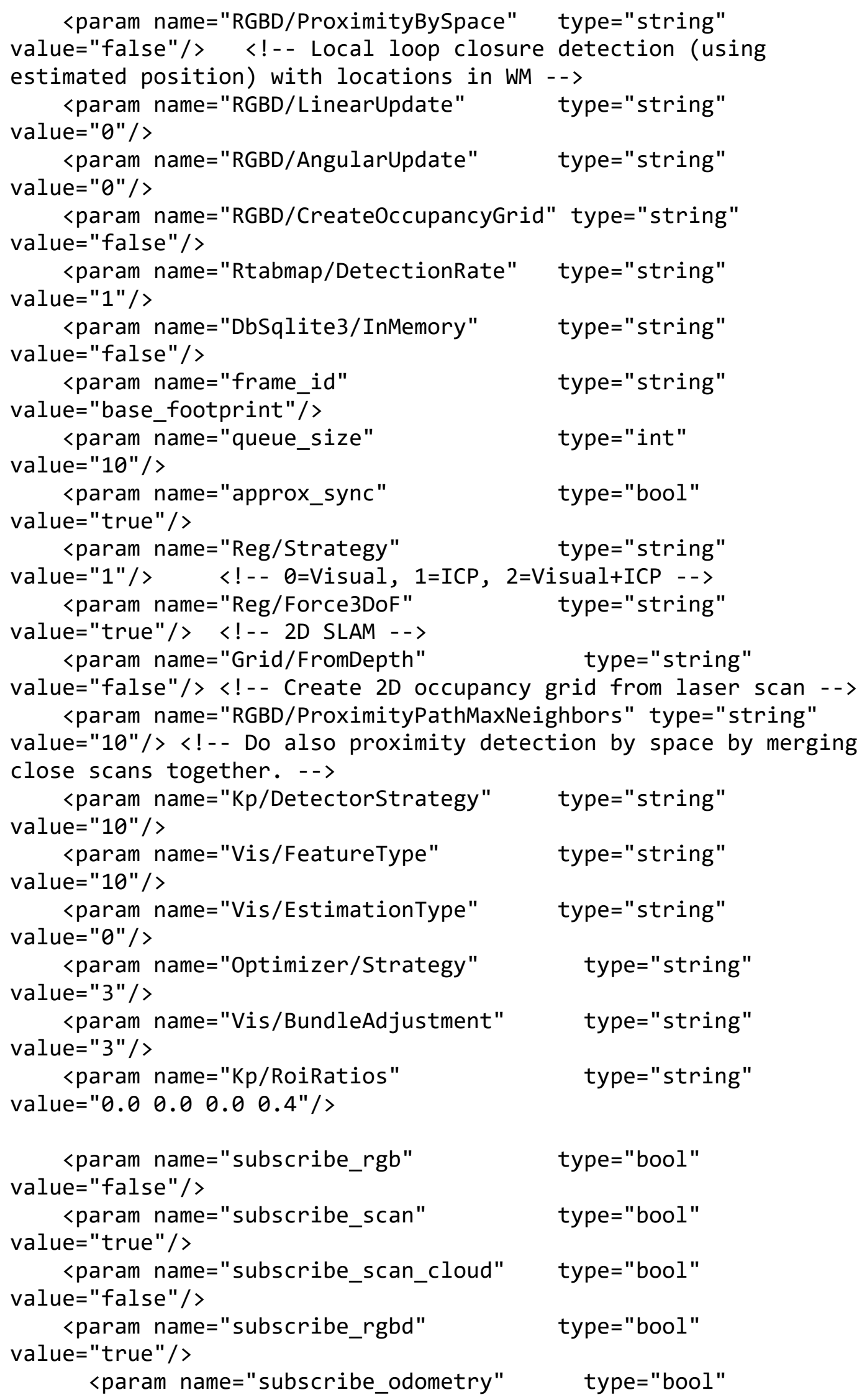




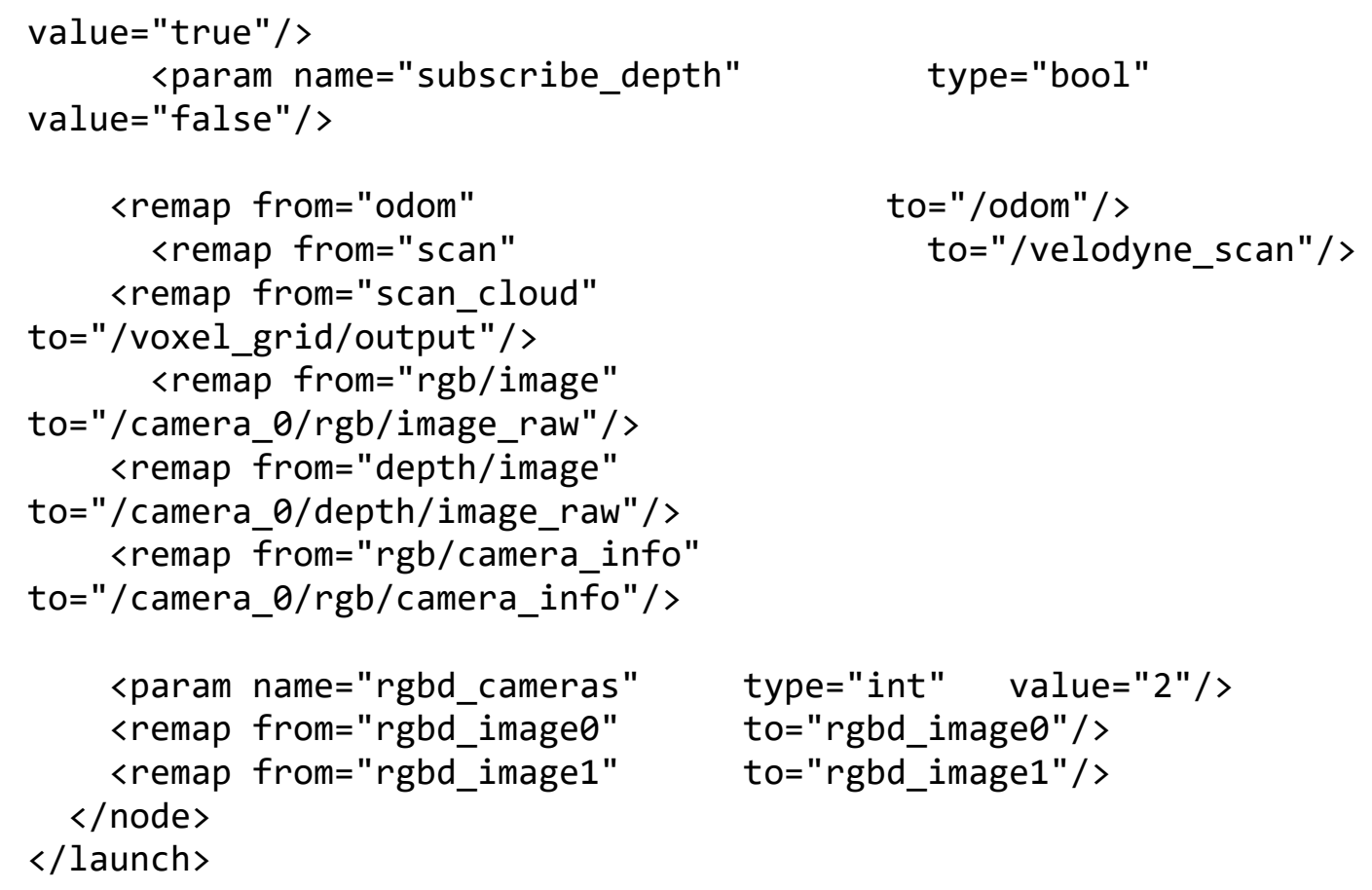

\section{tb3_mapper_params_online_sync.my_assembler}

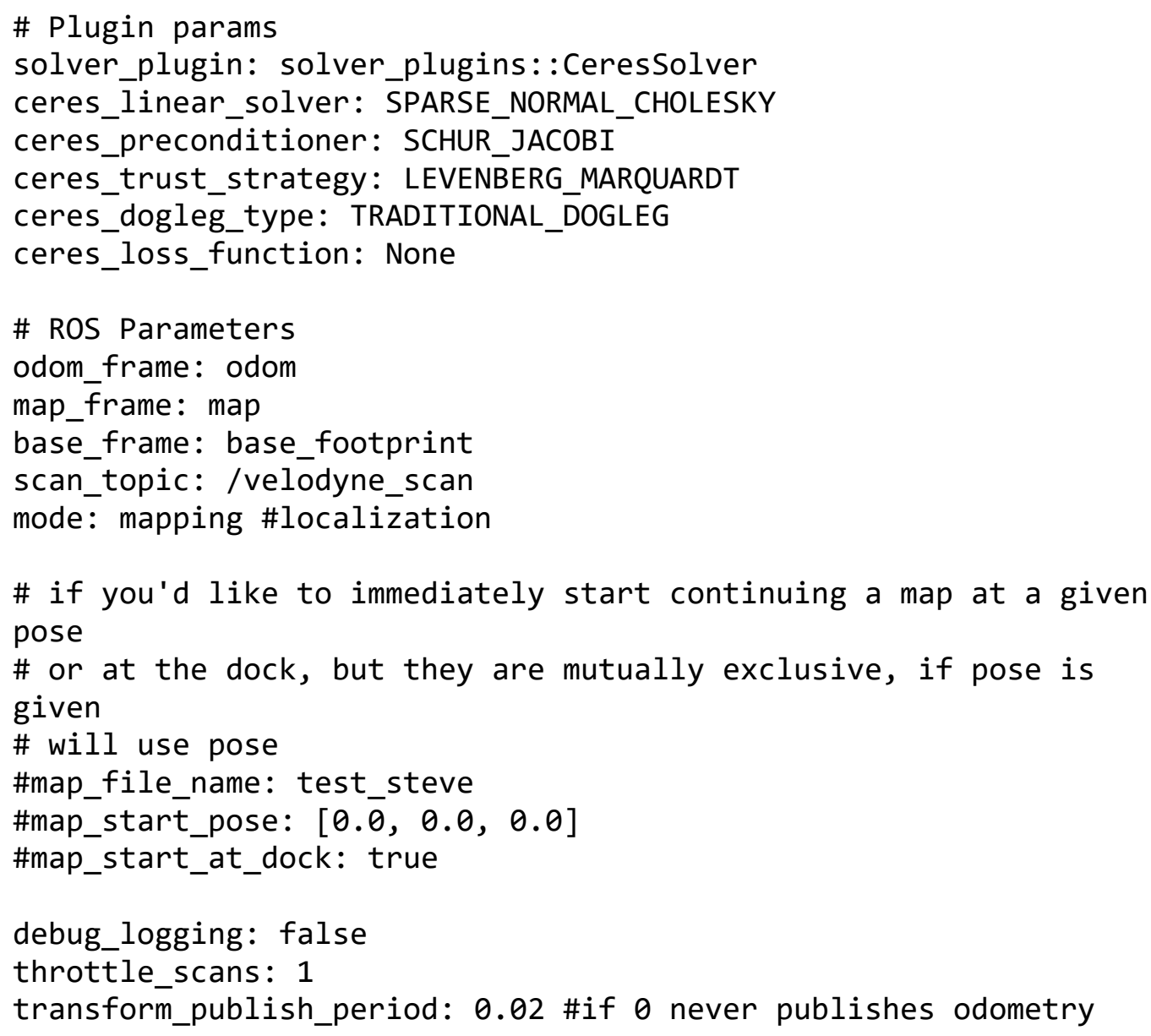


map_update_interval: 1.0

resolution: 0.05

max_laser_range: 100.0 \#for rastering images

minimum_time_interval: 0.5

transform_timeout: 0.2

tf_buffer_duration: 30 .

stack_size_to_use: 40000000 \#// program needs a larger stack size

to serialize large maps

enable_interactive_mode: true

\# General Parameters

use_scan_matching: true

use_scan_barycenter: true

minimum_travel_distance: 0.5

minimum_travel_heading: 0.5

scan_buffer_size: 10

scan_buffer_maximum_scan_distance: 10

link_match_minimum_response_fine: 0.1

link_scan_maximum_distance: 1.5

loop_search_maximum_distance: 3.0

do_loop_closing: true

loop_match_minimum_chain_size: 10

loop_match_maximum_variance_coarse: 3.0

loop_match_minimum_response_coarse: 0.35

loop_match_minimum_response_fine: 0.45

\# Correlation Parameters - Correlation Parameters

correlation_search_space_dimension: 0.5

correlation_search_space_resolution: 0.01

correlation_search_space_smear_deviation: 0.1

\# Correlation Parameters - Loop Closure Parameters

loop_search_space_dimension: 8.0

loop_search_space_resolution: 0.05

loop_search_space_smear_deviation: 0.03

\# Scan Matcher Parameters

distance_variance_penalty: 0.5

angle_variance_penalty: 1.0

fine_search_angle_offset: 0.00349

coarse_search_angle_offset: 0.349

coarse_angle_resolution: 0.0349

minimum_angle_enenalty: 0.9

minimum_distance_penalty: 0.5

use_response_expansion: true 


\section{Appendix C}

\section{tb3_move_base_slambox_STVL_eband.launch}

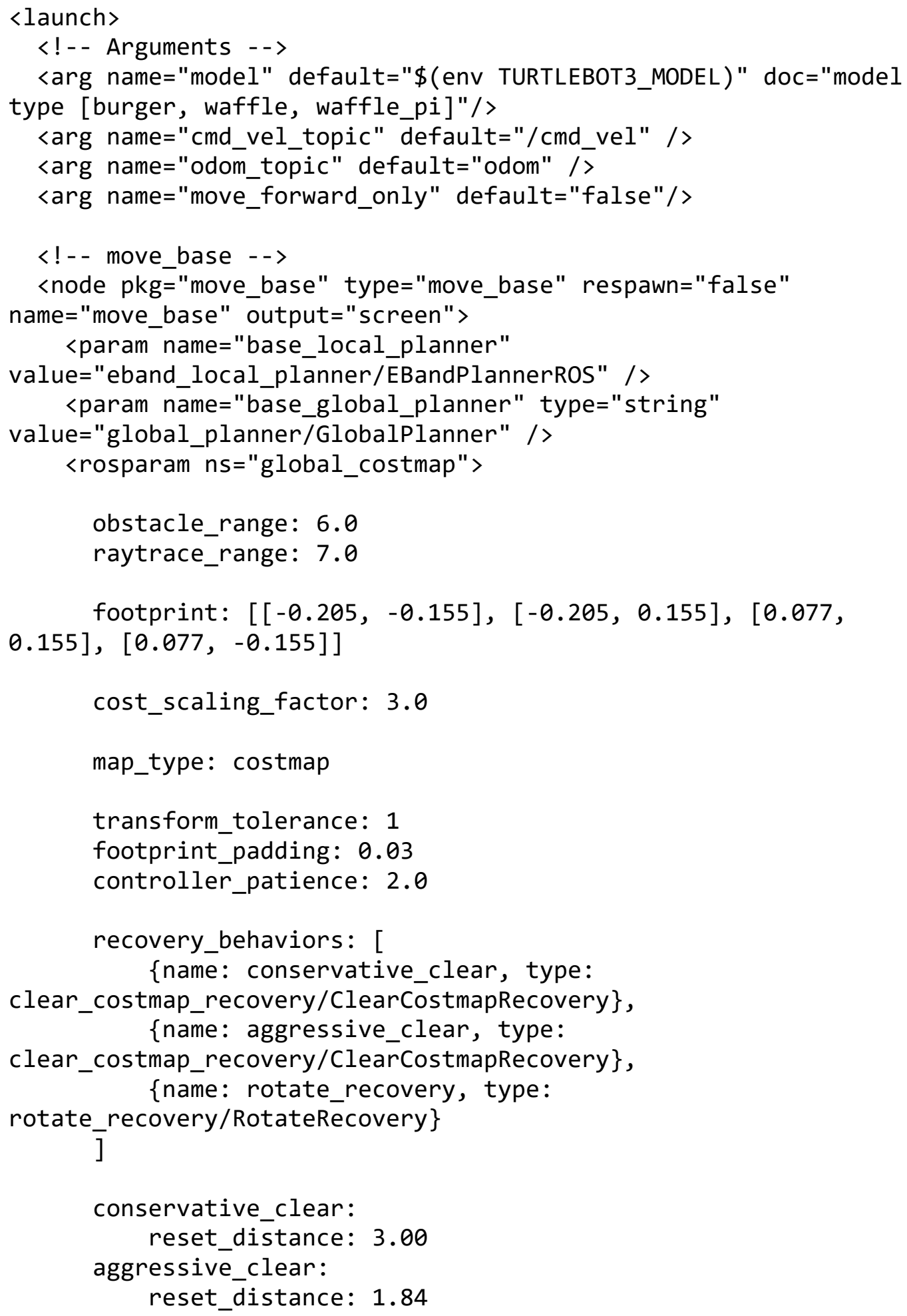




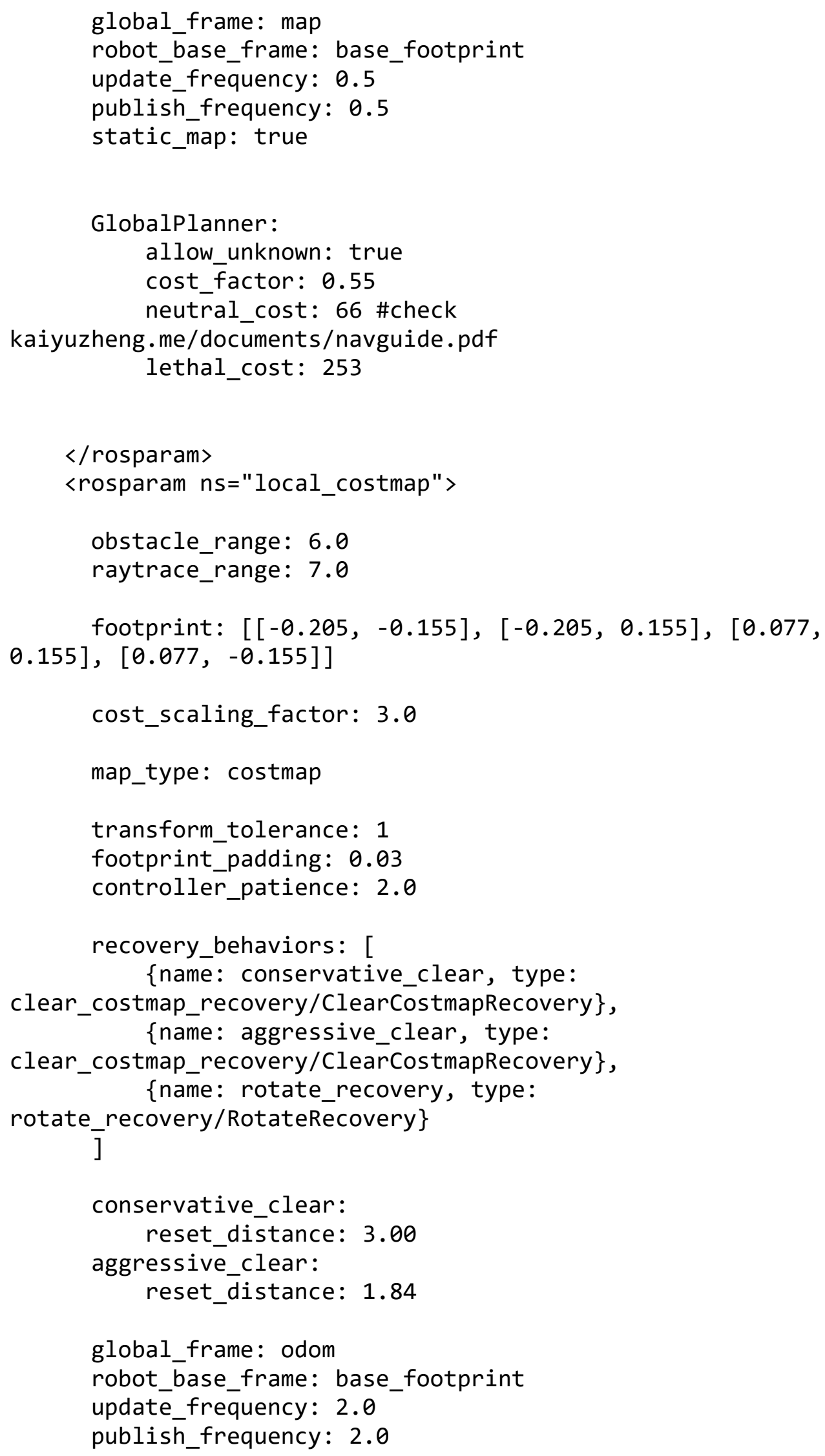




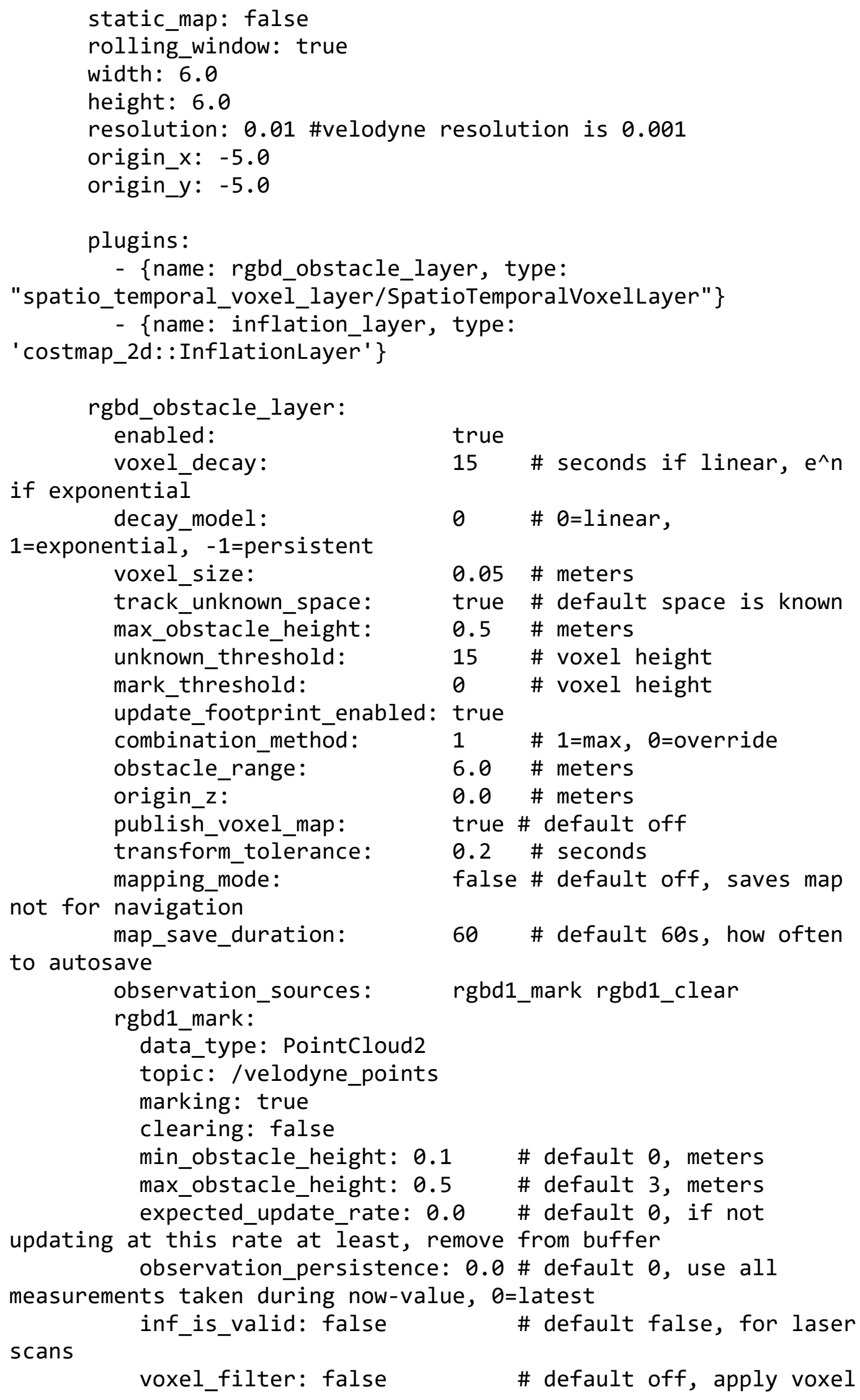


filter to sensor, recommend on

voxel_min_points: 0

points per voxe $\bar{l}$ for voxel filter

\# default 0 , minimum

clear_after_reading: true \# default false, clear the

buffer after the layer gets readings from it

rgbd1_clear:

enabled: true \#default true, can be

toggled on/off with associated service call

data_type: PointCloud2

topic: /velodyne_points

marking: false

clearing: true

max_z: 7.0 \# default 0 , meters

min_z: 0.2 \# default 10, meters

vertical_fov_angle: 0.523 \# default 0.7 , radians. For

3D lidars it's the symmetric fov about the planar axis.

0 , in meters

vertical_fov_padding: 0.05 \# 3D Lidar only. Default

horizontal_fov_angle: 6.29 \# 3D lidar scanners like

the VLP16 have 360 deg horizontal FOV.

decay_acceleration: $5.0 \quad \#$ default $0,1 / \mathrm{s}^{\wedge} 2$.

model_type: 1 \# default 0 , model type for

frustum. $\theta=$ depth camera, $1=3 \mathrm{~d}$ lidar like VLP16 or similar

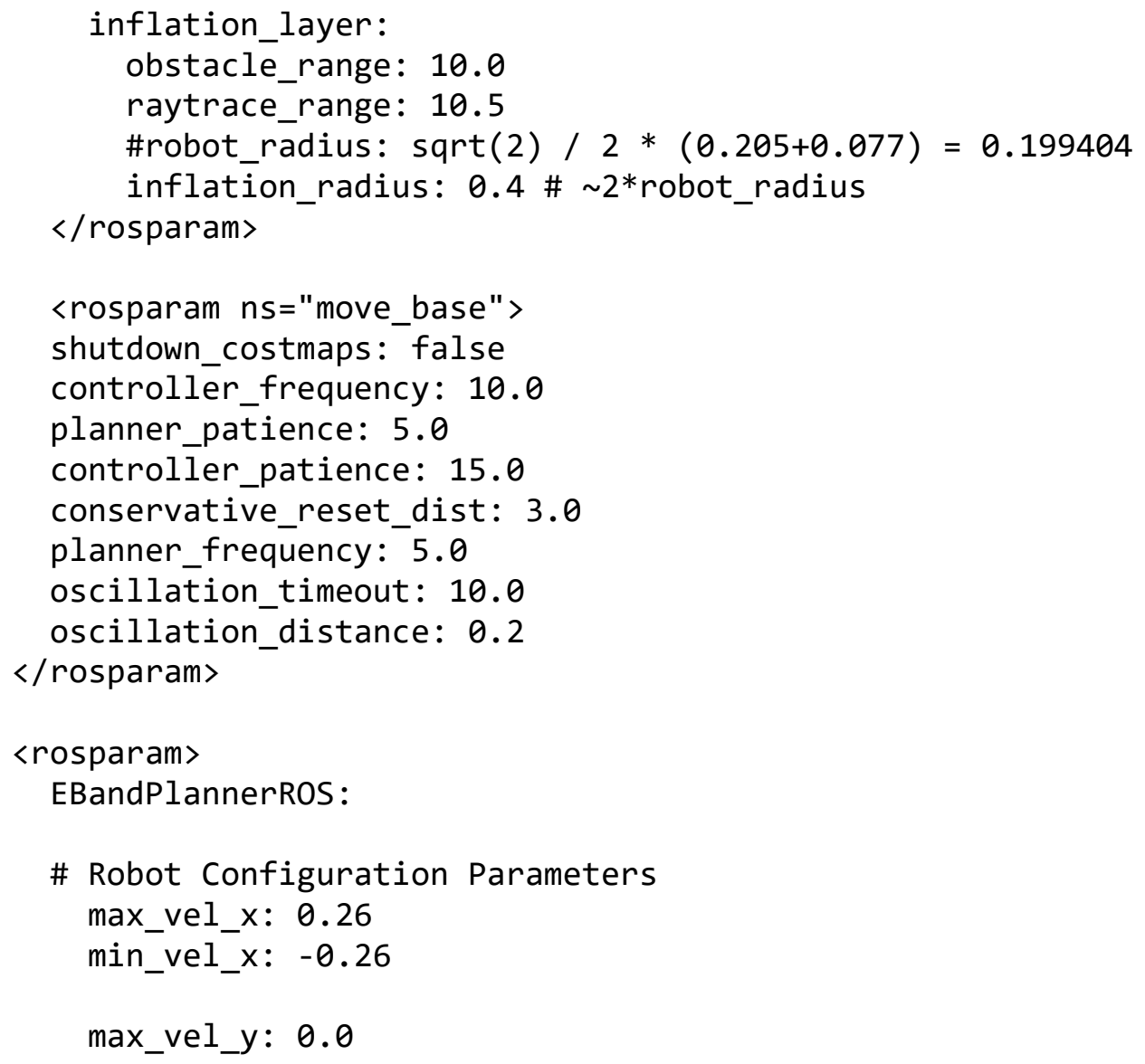




\section{Appendix D}

\section{explore.cpp}

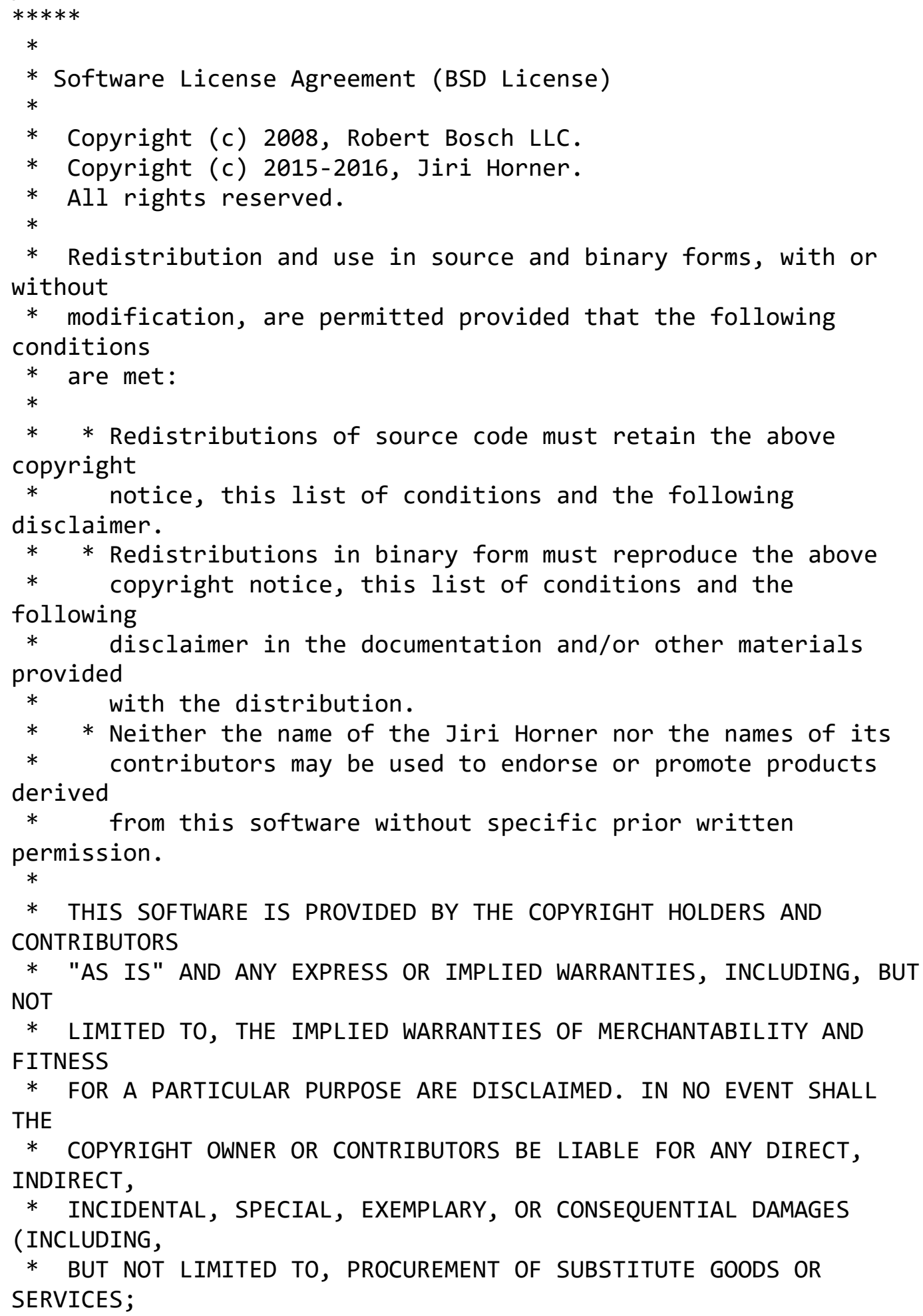


* LOSS OF USE, DATA, OR PROFITS; OR BUSINESS INTERRUPTION) HOWEVER

* CAUSED AND ON ANY THEORY OF LIABILITY, WHETHER IN CONTRACT, STRICT

* LIABILITY, OR TORT (INCLUDING NEGLIGENCE OR OTHERWISE) ARISING IN

* ANY WAY OUT OF THE USE OF THIS SOFTWARE, EVEN IF ADVISED OF THE

* POSSIBILITY OF SUCH DAMAGE.

$*$

$* * * * * * * * * * * * * * * * * * * * * * * * * * * * * * * * * * * * * * * * * * * * * * * * * * * * * * * * * * * * * * * * *$

$* * * * /$

\#include <explore/explore.h〉

\#include <thread>

inline static bool operator==(const geometry_msgs::Point\& one, const geometry_msgs::Point\& two)

\{

double $d x=$ one. $x$ - two. $x$;

double dy = one.y - two.y;

double dist $=\operatorname{sqrt}(d x * d x+d y * d y)$;

\}

return dist $<0.01$;

namespace explore

\{

Explore: : Explore( )

: private_nh_(" ")

, tf_listener_(ros: :Duration(10.0))

, costmap_client_(private_nh_, relative_nh_, \&tf_listener_)

, move_base_client_("move_base")

, prev_distance_( $\theta)$

\{

, last_markers_count_( $\theta)$

double timeout;

double min_frontier_size;

private_nh_.param("planner_frequency", planner_frequency_,

$1.0)$;

private_nh_.param("progress_timeout", timeout, 30.0);

progress_timeout_ = ros: :Duration(timeout);

private_nh_.param("visualize", visualize_, false);

private_nh_.param("potential_scale", potential_scale_, 1e-3);

private_nh_.param("orientation_scale", orientation_scale_,

$0.0)$;

private_nh_.param("gain_scale", gain_scale_, 1.0);

private_nh_.param("min_frontier_size", min_frontier_size, 0.5); 


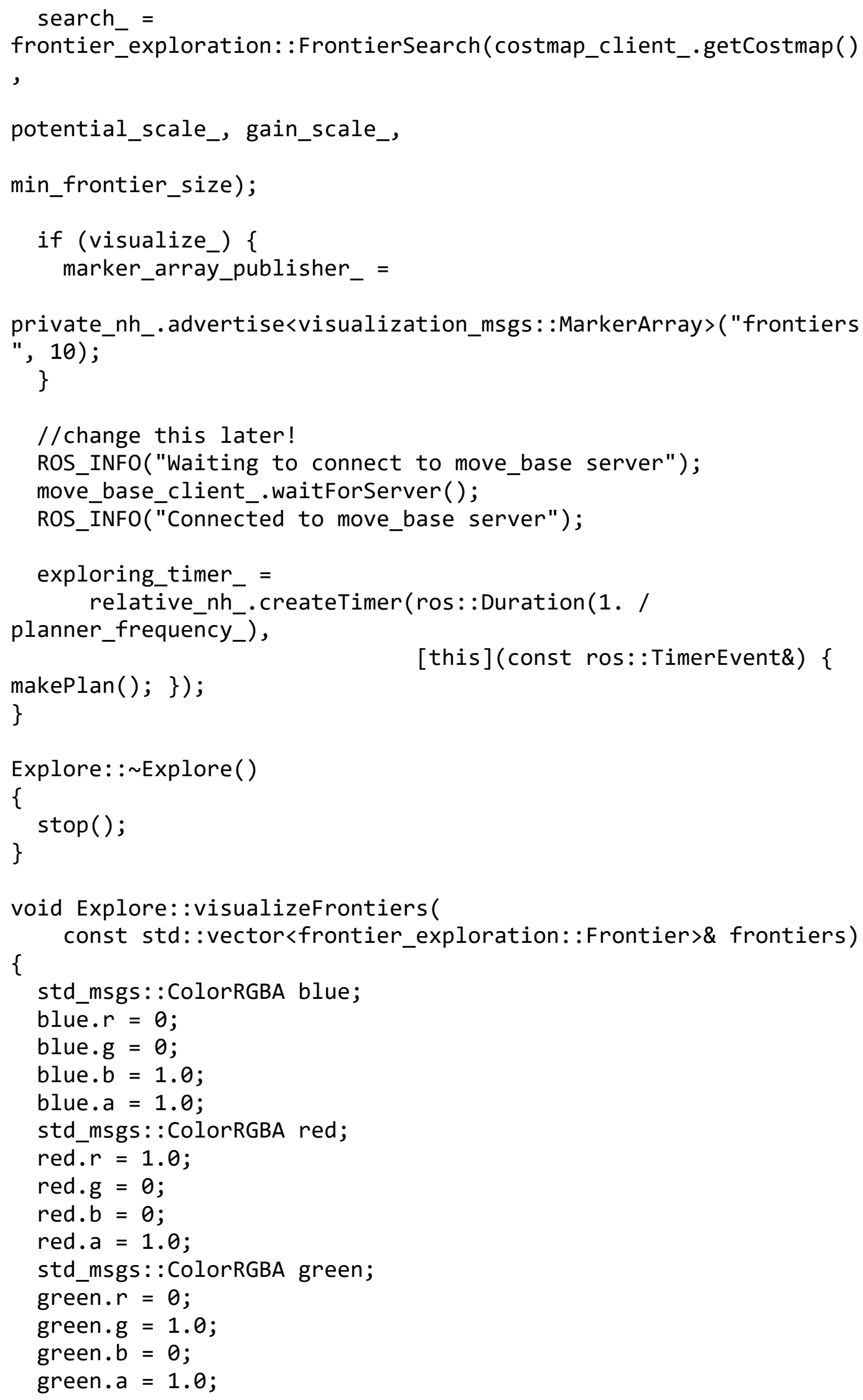


ROS_DEBUG("visualising \%lu frontiers", frontiers.size()); visualization_msgs: :MarkerArray markers_msg;

std: : vector $\langle$ visualization_msgs: :Marker $>\&$ markers $=$ markers_msg.markers;

visualization_msgs::Marker m;

$\mathrm{m}$. header.frame_id $=$ costmap_client_.getGlobalFrameID () ;

m. header. stamp = ros: :Time: :now () ;

m.ns = "frontiers";

m. scale. $x=1.0$;

m.scale.y $=1.0$;

m. scale. $z=1.0$;

m. color. $r=0$;

m. color.g $=0$;

m. color. $b=255$;

m. color. a $=255$;

// lives forever

m.lifetime $=$ ros: $:$ Duration $(\theta)$;

m. frame_locked = true;

// weighted frontiers are always sorted double min_cost $=$ frontiers.empty () ? 0 . :

frontiers.front (). cost;

m.action = visualization_msgs: $:$ Marker: :ADD;

size_t id $=0$;

for (auto\& frontier : frontiers) \{

m.type = visualization_msgs: :Marker::POINTS;

m.id = int (id);

m.pose.position $=\{\}$;

m. scale. $x=0.1$;

m.scale.y $=0.1$;

m.scale.z $=0.1$;

m.points = frontier. points;

if (goalonBlacklist(frontier.centroid)) \{

$\mathrm{m}$. color = red;

\} else \{

\}

m.color = blue;

markers.push_back $(\mathrm{m})$;

++id;

m.type = visualization_msgs::Marker::SPHERE;

m.id = int (id);

m. pose. position = frontier.initial;

// scale frontier according to its cost (costier frontiers

will be smaller)

double scale = std::min(std::abs(min_cost $* 0.4 /$

frontier.cost), 0.5 );

m.scale.x = scale; 


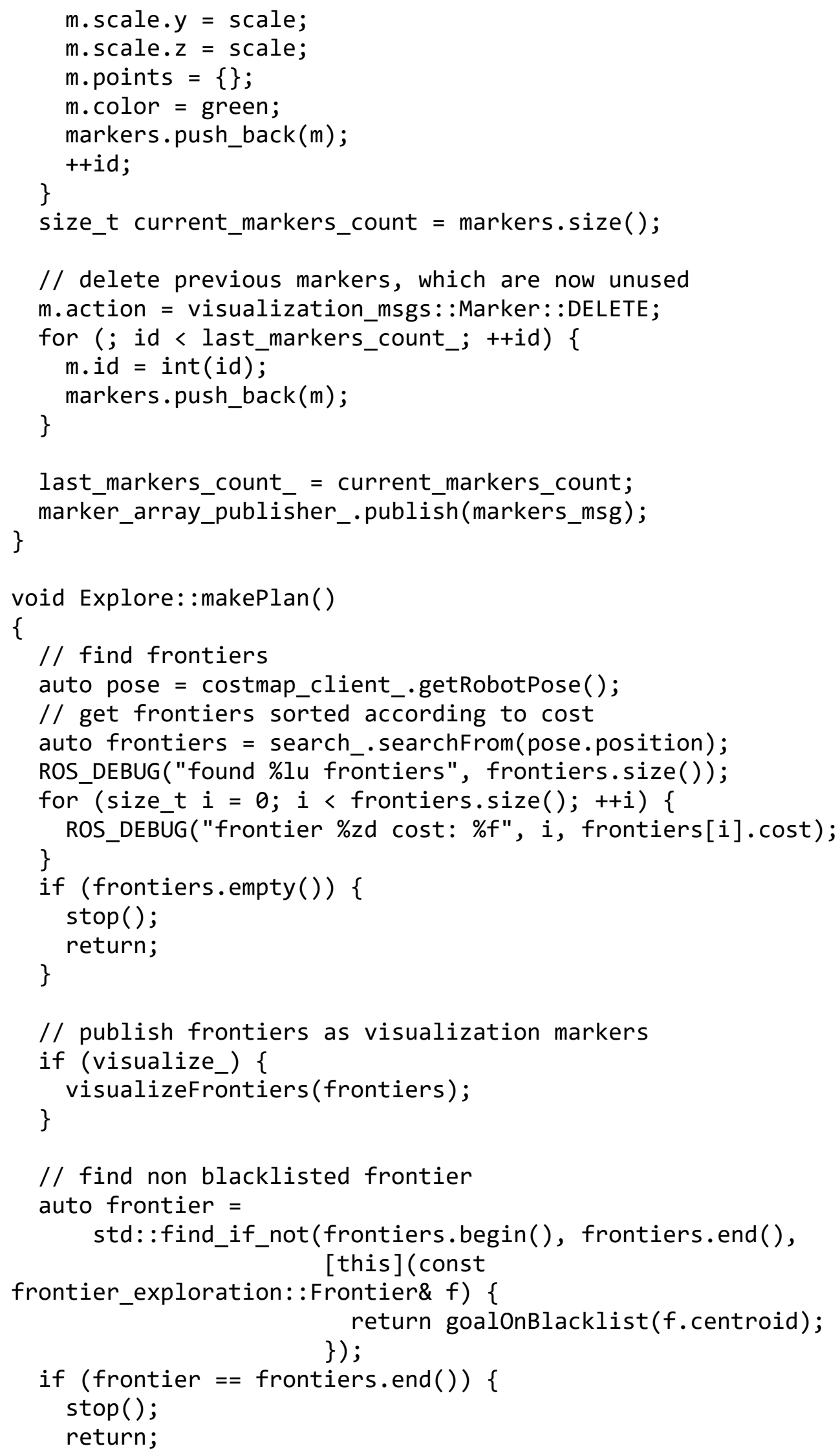




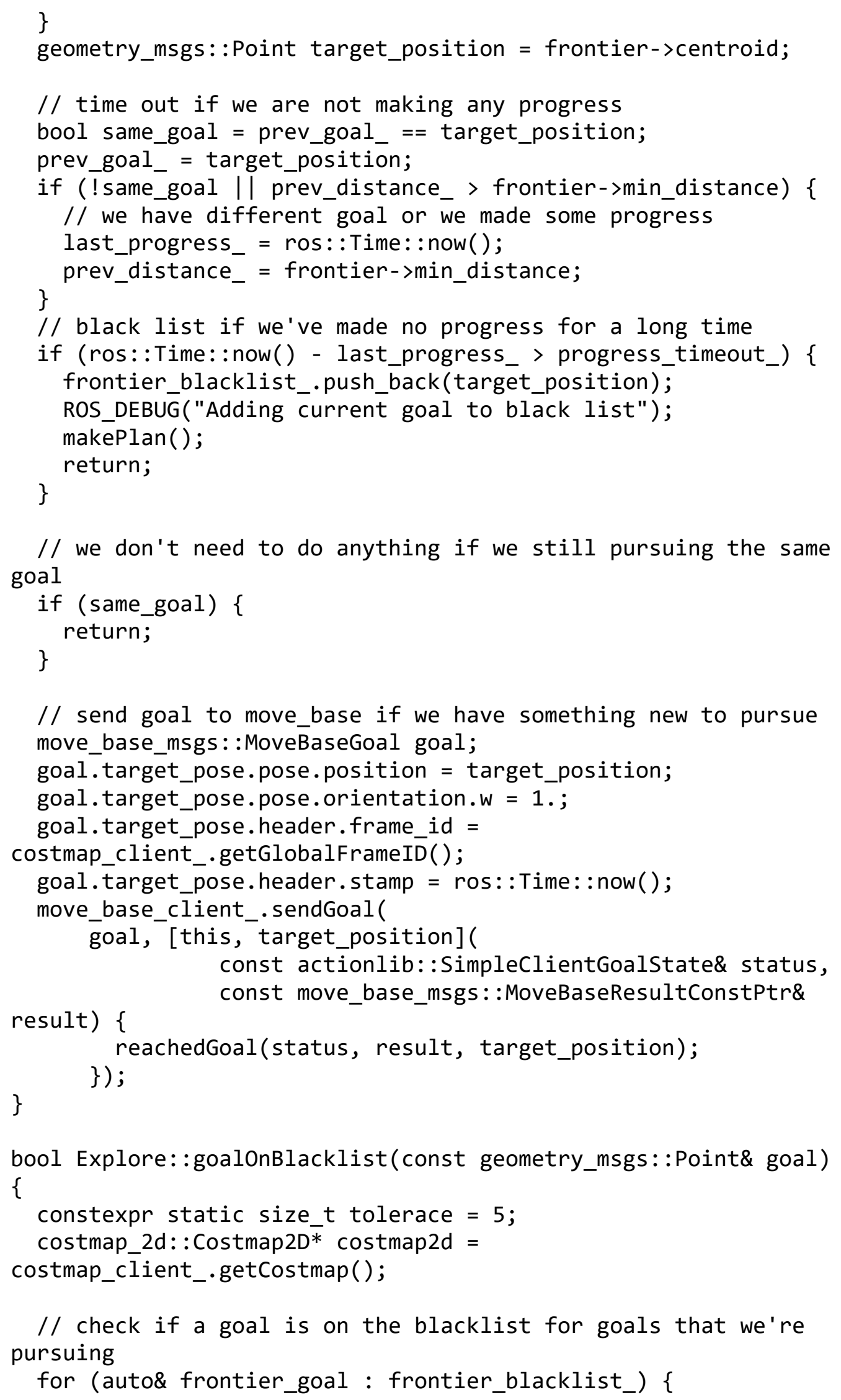




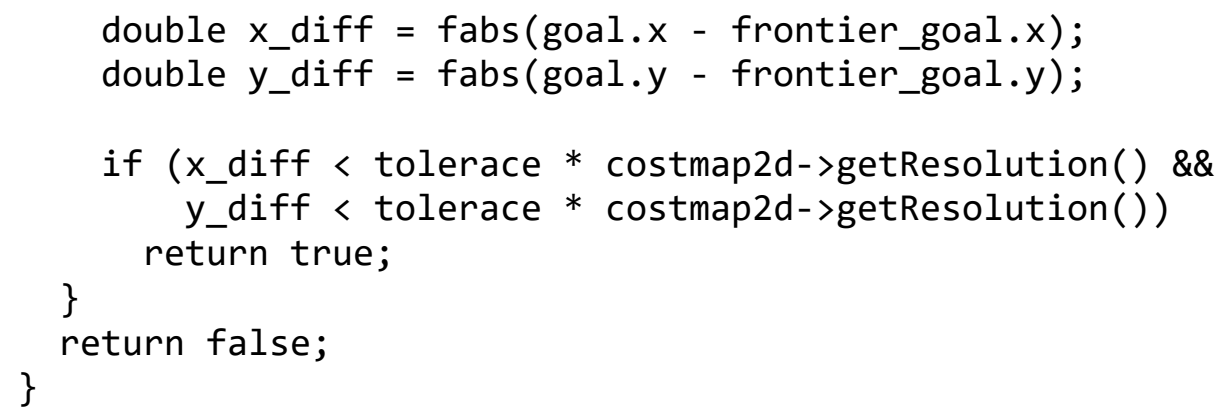




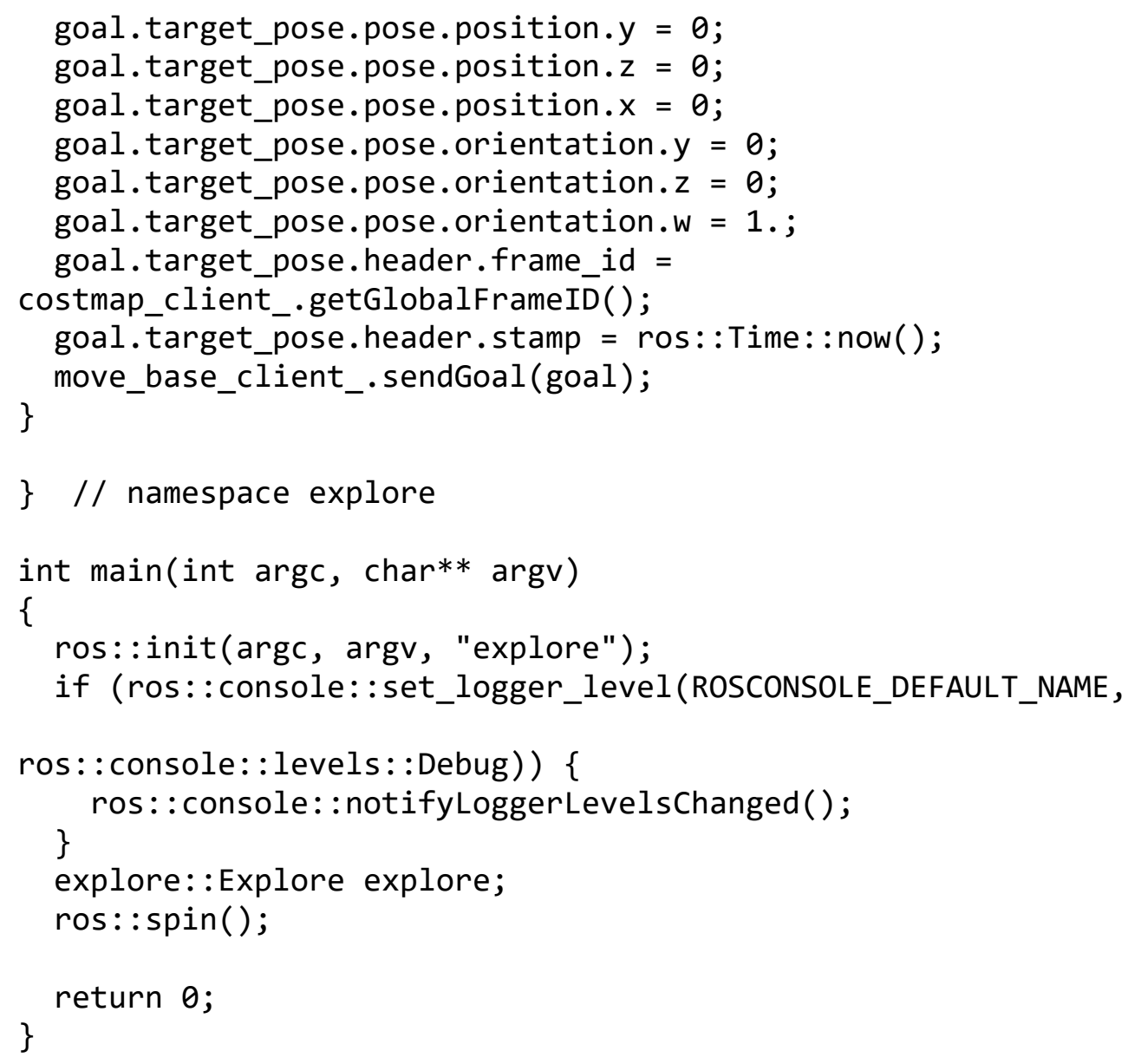

\section{frontier_search.cpp}

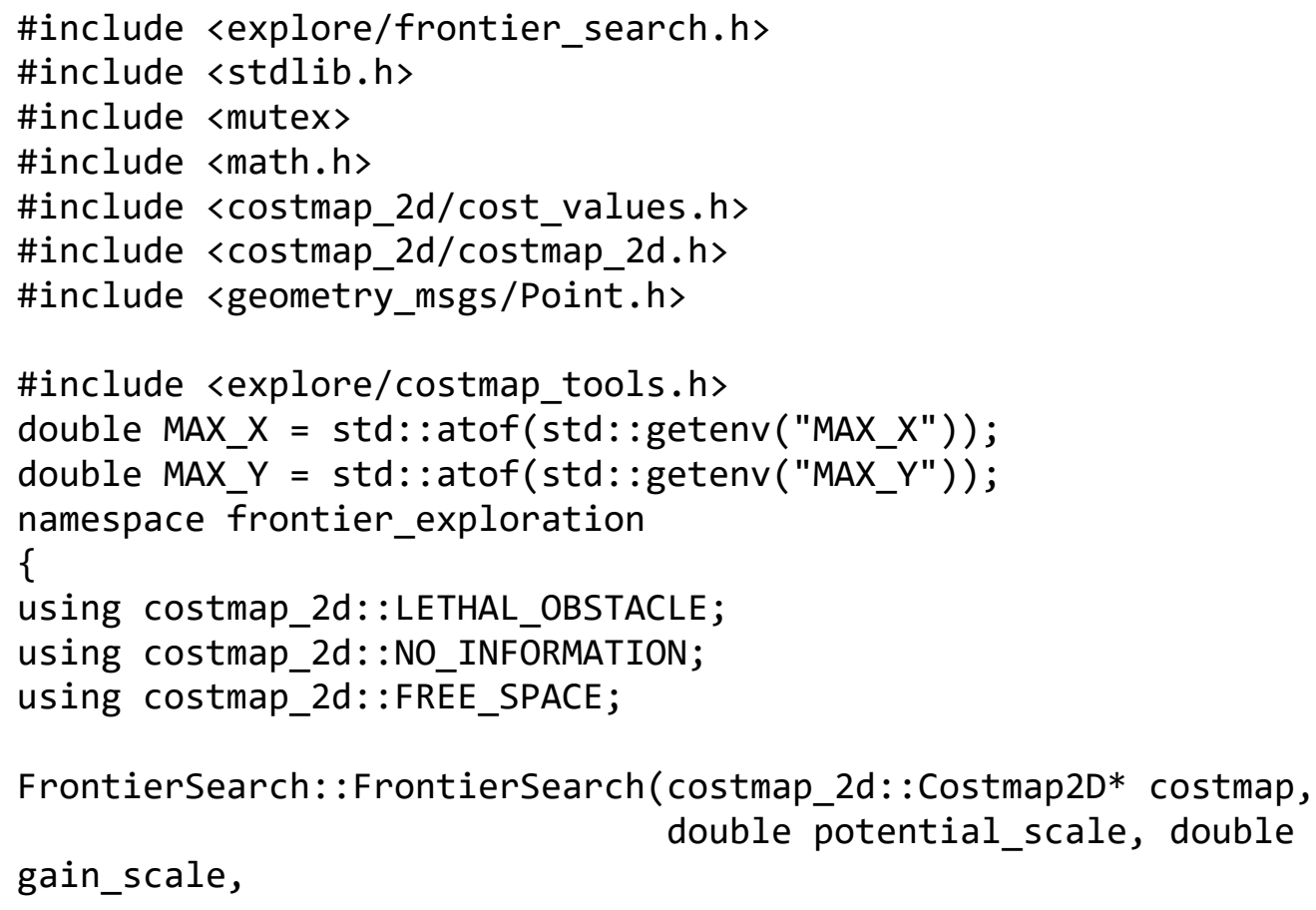


: costmap_(costmap)

double min_frontier_size)

, potential_scale_(potential_scale)

, gain_scale_(gain_scale)

\{

, min_frontier_size_(min_frontier_size)

\}

std: : vector<Frontier>

FrontierSearch::searchFrom(geometry_msgs::Point position)

\{

std: : vector<Frontier $>$ frontier_list;

// Sanity check that robot is inside costmap bounds before searching

unsigned int $m x$, my;

if (! costmap_->worldToMap(position.x, position.y, mx, my)) \{

ROS_ERROR("Robot out of costmap bounds, cannot search for frontiers");

\}

return frontier_list;

// make sure map is consistent and locked for duration of search

std: :lock_guard<costmap_2d::Costmap2D: :mutex_t>

lock $\left(*\left(\operatorname{costmap} \_>\right.\right.$getMutex ()$\left.)\right)$;

map_ = costmap_->getCharMap ( );

size_x_ = costmap_- >getSizeInCellsX ( );

size_y_ $=$ costmap_- $>$ getSizeInCellsY () ;

// initialize flag arrays to keep track of visited and frontier cells

std: :vector<bool> frontier_flag(size_x_* size_y_, false);

std: :vector $\langle$ bool> visited_flag(size_x_* size_y_, false);

// initialize breadth first search

std: :queue<unsigned int> bfs;

// find closest clear cell to start search

unsigned int clear, pos = costmap_->getIndex (mx, my);

if (nearestCell(clear, pos, FREE_SPACE, *costmap_)) \{

bfs.push(clear);

\} else \{

bfs.push(pos);

\}

ROS_WARN("Could not find nearby clear cell to start search");

visited_flag[bfs.front ()] = true;

while (!bfs.empty ()) \{

unsigned int idx = bfs.front (); 


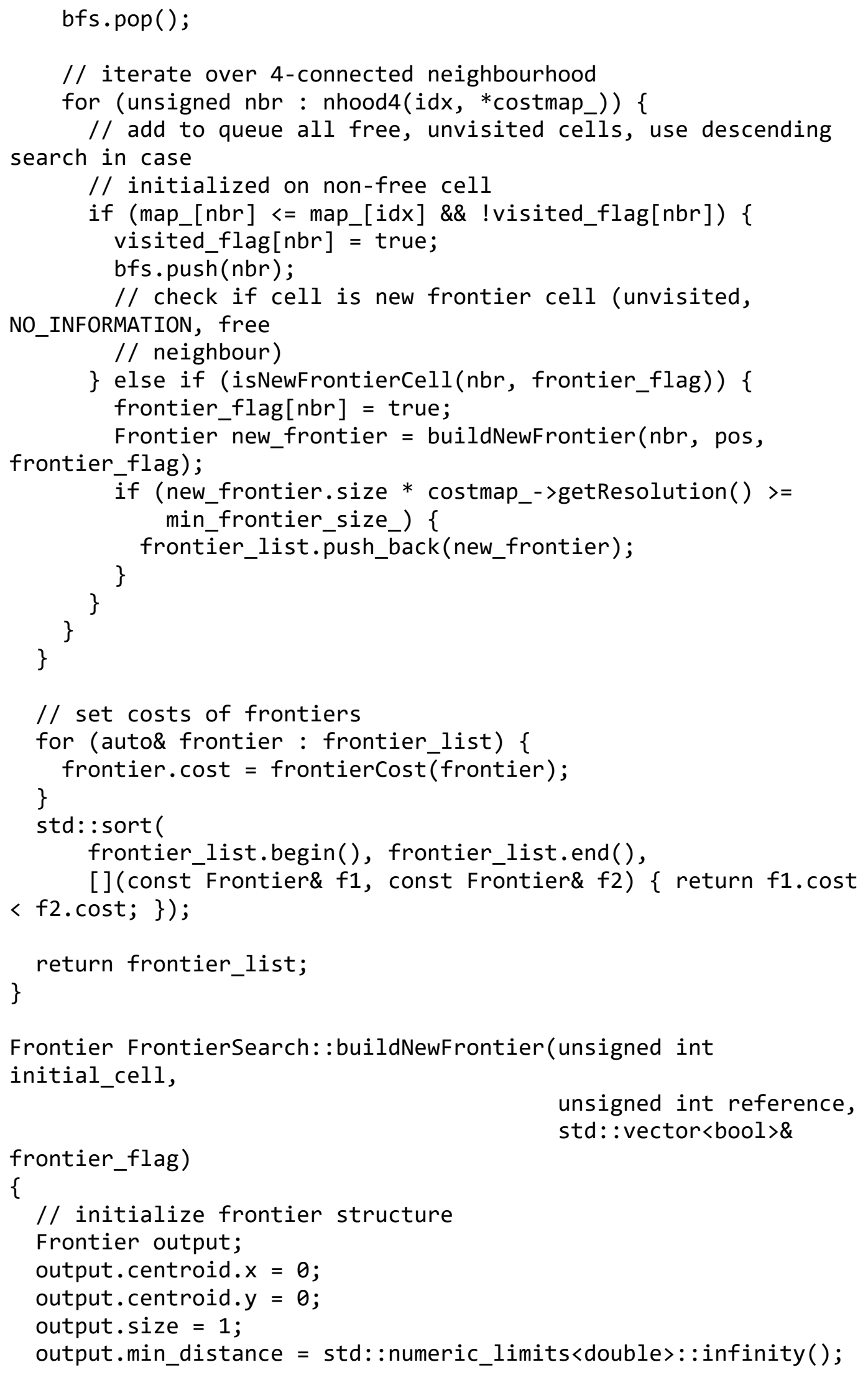


// record initial contact point for frontier unsigned int ix, iy; costmap_->indexToCells(initial_cell, ix, iy); costmap_->mapToWorld(ix, iy, output.initial.x, output.initial.y);

// push initial gridcell onto queue std: :queue<unsigned int> bfs;

bfs.push(initial_cell);

// cache reference position in world coords unsigned int $r x$, ry; double reference_x, reference_y; costmap_->indexToCells(reference, rx, ry); costmap_->mapToWorld(rx, ry, reference_x, reference_y);

while (!bfs.empty ()) \{ unsigned int idx = bfs.front ();

bfs.pop();

// try adding cells in 8-connected neighborhood to frontier for (unsigned int nbr : nhood8(idx, *costmap_)) \{

// check if neighbour is a potential frontier cell

if (isNewFrontierCell(nbr, frontier_flag)) \{

// mark cell as frontier frontier_flag[nbr] = true;

unsigned int $\mathrm{mx}$, my;

double wx, wy; costmap_->indexToCells(nbr, mx, my); costmap_->mapToWorld(mx, my, wx, wy);

geometry_msgs::Point point;

point. $x=w x$;

point.y = wy;

output.points.push_back(point);

// update frontier size

output.size++;

// update centroid of frontier

output. centroid. $x+=w x$;

output. centroid.y $+=$ wy;

// determine frontier's distance from robot, going by

closest gridcell

// to robot

double distance $=\operatorname{sqrt}($ pow $(($ double $($ reference_x $)$ double $(w x)), 2.0)+$

double(wy)), 2.0));

$$
\text { pow((double(reference_y) - }
$$




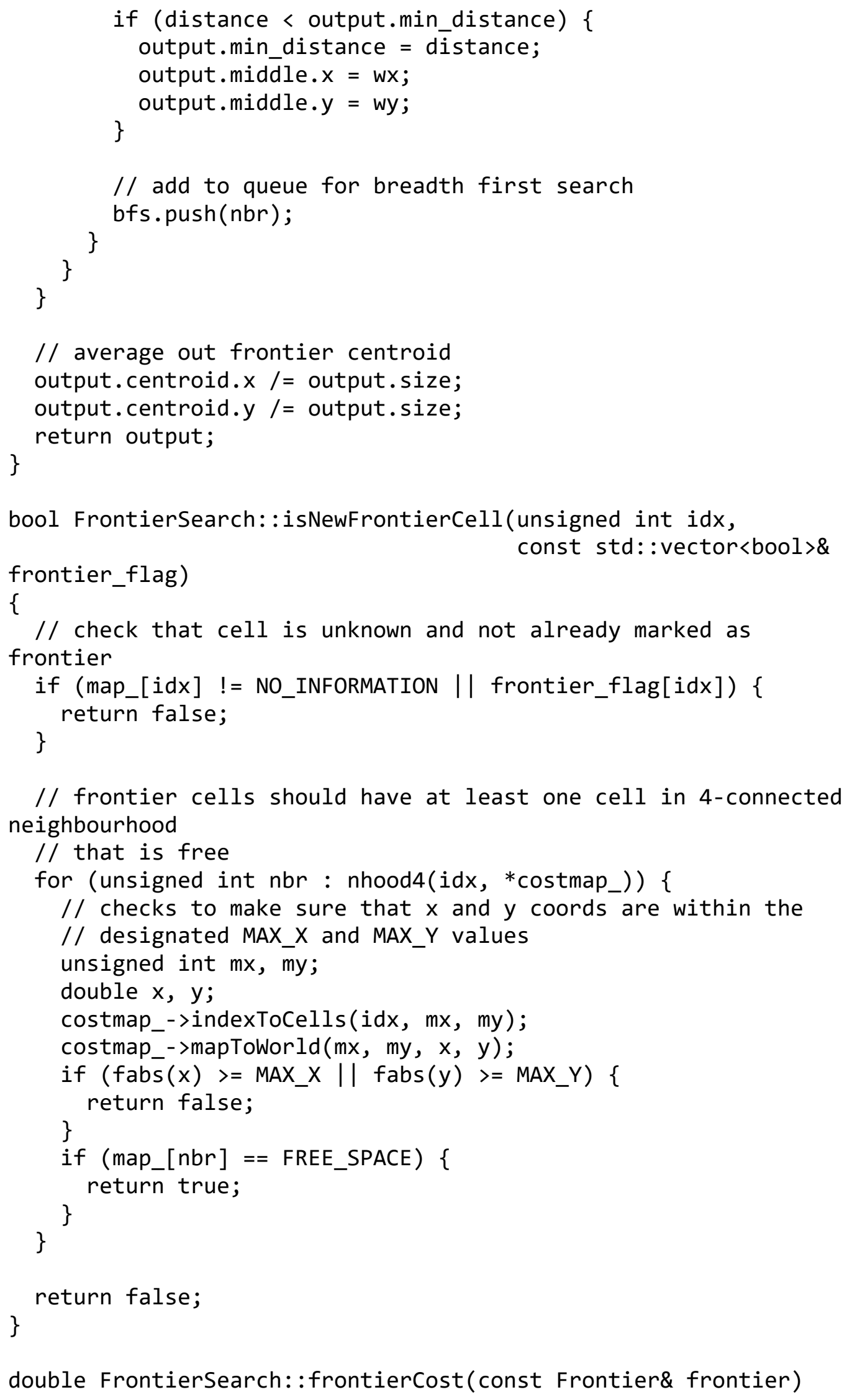




\section{\{}

return (potential_scale_ ${ }^{*}$ frontier.min_distance $*$ costmap_->getResolution())

(gain_scale_ $*$ frontier.size $*$ costmap_$>$ getResolution( ));

\}
\} 


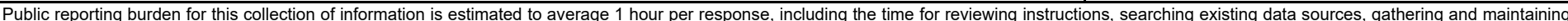

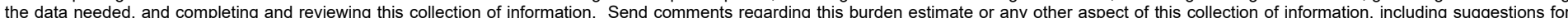

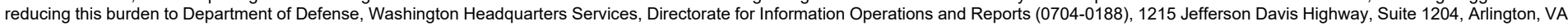

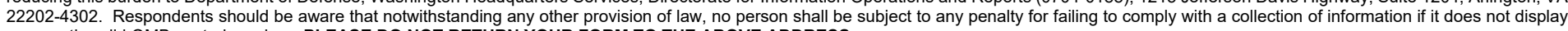
a currently valid OMB control number. PLEASE DO NOT RETURN YOUR FORM TO THE ABOVE ADDRESS.

\begin{tabular}{|l|l|l|} 
1. REPORT DATE (DD-MM-YYYY) & 2. REPORT TYPE & 3. DATES COVERED (FrOm - To)
\end{tabular}

September 2021 Final

\section{TITLE AND SUBTITLE}

5a. CONTRACT NUMBER

Autonomous Navigation and Mapping in a Simulated Environment

5b. GRANT NUMBER

5c. PROGRAM ELEMENT NUMBER 622146

6. AUTHOR(S)

5d. PROJECT NUMBER

AT9

Benjamin Christie, Osama Ennasr, and Garry Glaspell

5e. TASK NUMBER

01

5f. WORK UNIT NUMBER

\section{PERFORMING ORGANIZATION NAME(S) AND ADDRESS(ES)}

8. PERFORMING ORGANIZATION REPORT NUMBER

Geospatial Research Laboratory

U.S. Army Engineer Research and Development Center

ERDC/GRL TR-21-5

7701 Telegraph Road

Alexandria, VA 22315

9. SPONSORING / MONITORING AGENCY NAME(S) AND ADDRESS(ES)

10. SPONSOR/MONITOR'S ACRONYM(S)

U.S. Army Corps of Engineers

Washington, DC 20314-1000

11. SPONSOR/MONITOR'S REPORT NUMBER(S)

\section{DISTRIBUTION / AVAILABILITY STATEMENT}

Approved for public release; distribution is unlimited.

\section{SUPPLEMENTARY NOTES}

\section{ABSTRACT}

Unknown Environment Exploration (UEE) with an Unmanned Ground Vehicle (UGV) is extremely challenging. This report investigates a frontier exploration approach, in simulation, that leverages Simultaneous Localization And Mapping (SLAM) to efficiently explore unknown areas by finding navigable routes. The solution utilizes a diverse sensor payload that includes wheel encoders, three-dimensional (3-D) LIDAR, and Red, Green, Blue and Depth (RGBD) cameras. The main goal of this effort is to leverage frontier-based exploration with a UGV to produce a 3-D map (up to $10 \mathrm{~cm}$ resolution). The solution provided leverages the Robot Operating System (ROS).

\section{SUBJECT TERMS}

Testing--Simulation methods

Autonomous robots--Navigation

\section{SECURITY CLASSIFICATION OF:}

\section{a. REPORT}

Unclassified

\section{b. ABSTRACT}

Unclassified

\section{c. THIS PAGE}

Unclassified
17. LIMITATION OF ABSTRACT

SAR
18. NUMBER OF PAGES

70 19a. NAME OF RESPONSIBLE PERSON

19b. TELEPHONE NUMBER (include area code) 